This is the post print version of the article, which has been published in Journal of algebra. 2018, 516 , 437-470. https://doi.org/10.1016/j.jalgebra.2018.09.016.

\title{
A FORMULA FOR JUMPING NUMBERS IN A TWO-DIMENSIONAL REGULAR LOCAL RING
}

\author{
EERO HYRY AND TARMO JÄRVILEHTO
}

\begin{abstract}
In this article we give an explicit formula for the jumping numbers of an ideal of finite colenght in a two-dimensional regular local ring with an algebraically closed residue field. For this purpose, we associate a certain numerical semigroup to each vertex of the dual graph of a log-resolution of the ideal.
\end{abstract}

\section{INTRODUCTION}

Jumping numbers measure the complexity of the singularities of a closed subscheme of a variety. They are defined in terms of multiplier ideals of the subscheme. Multiplier ideals form a nested sequence of ideals parametrized by rational numbers. The values of the parameter where the multiplier ideal changes are called jumping numbers. For a simple complete ideal in a local ring of a closed point on a smooth surface an explicit formula has been provided by Järvilehto in [8], which is based on the dissertation [7]. This result applies also to jumping numbers of an analytically irreducible plane curve. The purpose of this article is to generalize this formula to any complete ideal.

Besides [8], jumping numbers of simple complete ideals or analytically irreducible plane curves have been independently investigated by several people (see, e. g., [13, [12, [15] and 44). In a local ring at a rational singularity of a complex surface, Tucker presented in [16] an algorithm to compute the set of jumping numbers of any ideal. Recently, Alberich-Carramiñana, Montaner and Dachs-Cadefau gave in [1] another algoritm for this purpose. But even in dimension two finding a closed formula for the general case has turned out to be difficult. Kuwata calculated in [9] the smallest jumping number, the so called log-canonical threshold, for a reduced plane curve with two branches. Galindo, Hernando and Monserrat succeeded in [5] to generalize this to any number of branches.

Jumping numbers are defined by using an embedded resolution of the subscheme. They depend on the exceptional divisors appearing in the resolution. We therefore look at the dual graph of the resolution. Recall that the vertices of the dual graph correspond to exceptional divisors and two vertices are connected by an edge if the exceptional divisors in question intersect. To each vertex, we will attach a certain semigroup. We will then describe jumping numbers in terms of these semigroups. In defining the semigroups we use Zariski exponents of the valuations associated to the exceptional divisors. 
To explain this in more detail, let $\mathfrak{a}$ be a complete ideal of finite colength in a two-dimensional regular local ring $R$ having an algebraically closed residue field. Let $X \longrightarrow$ Spec $R$ be a $\log$ resolution of the pair $(R, \mathfrak{a})$. Let $E_{1}, \ldots, E_{N}$ be the exceptional divisors. Let $\Gamma$ be the dual graph of $X$. Two vertices $\gamma$ and $\eta$ are called adjacent, denoted by $\gamma \sim \eta$, if the corresponding exceptional divisors $E_{\gamma}$ and $E_{\eta}$ intersect. The valence $v_{\Gamma}(\mu)$ of a vertex $\mu$ means the number of vertices adjacent to it. A vertex with valence at most one is called an end whereas a vertex of valence at least three is a star. Let $v_{1}, \ldots, v_{N}$ be the discrete valuations and $\mathfrak{p}_{1}, \ldots, \mathfrak{p}_{N}$ the simple ideals corresponding to $E_{1}, \ldots, E_{N}$, respectively. Set $V_{\mu, \nu}=v_{\mu}\left(\mathfrak{p}_{\nu}\right)$ for all $\mu, \nu=1, \ldots, N$. The Zariski exponents are the numbers $V_{\mu, \tau}$, where $\tau$ is end. Let $S^{\mu}$ denote the submonoid of $\mathbb{N}$ generated by $V_{\mu, \mu}$ and the numbers

$$
s_{\nu}^{\mu}:=\operatorname{gcd}\left\{V_{\mu, \tau} \mid v_{\Gamma}(\tau)=1 \text { and } \tau \in \Gamma_{\nu}^{\mu}\right\},
$$

where $\Gamma_{\nu}^{\mu}$ is the branch emanating from $\mu$ towards $\nu$, i. e., the maximal connected subgraph of $\Gamma$ containing $\nu$ but not $\mu$. We will show that $S^{\mu}$ is a numerical semigroup generated by at most two elements (see Remark 22).

Recall that a divisor $F=f_{1} E_{1}+\ldots+f_{N} E_{N}$ on $X$ is called antinef if $F \cdot E_{\gamma} \leq 0$ for all $\gamma=1, \ldots, N$, where $F \cdot E_{\gamma}$ is the intersection product. Let $\left\{\widehat{E}_{1}, \ldots, \widehat{E}_{N}\right\}$, where $E_{\mu} \cdot \widehat{E}_{\nu}=-\delta_{\mu, \nu}$, denote the dual basis of $\left\{E_{1}, \ldots, E_{N}\right\}$. Then $F=\widehat{f}_{1} \widehat{E}_{1}+\ldots+\widehat{f}_{N} \widehat{E}_{N}$ is antinef if and only if $\widehat{f}_{i} \geq 0$ for all $i=1, \ldots, N$. We call the numbers $\widehat{f}_{1}, \ldots, \widehat{f}_{N}$ as the factors of $F$.

We make use of the observation made in [6] that jumping numbers of $\mathfrak{a}$ can be parametrized by the antinef divisors. More precisely, the jumping number corresponding to an antinef divisor $F$ is

$$
\xi_{F}:=\min _{\gamma} \frac{f_{\gamma}+k_{\gamma}+1}{d_{\gamma}},
$$

where $D=d_{1} E_{1}+\ldots+d_{N} E_{N}$ is the divisor on $X$ such that $\mathcal{O}_{X}(-D)=\mathfrak{a} \mathcal{O}_{X}$, and $K=k_{1} E_{1}+\ldots+k_{N} E_{N}$ denotes the canonical divisor. We say that $\xi$ is a jumping number supported at a vertex $\mu$ if $\xi=\xi_{F}$ for some antinef divisor $F$ with

$$
\xi_{F}=\frac{f_{\mu}+k_{\mu}+1}{d_{\mu}} .
$$

We fix a vertex $\mu$ and concentrate on the set $\mathcal{H}_{\mu}^{\mathfrak{a}}$ of jumping numbers supported at $\mu$.

Our main result, Theorem 23, yields a formula for the set of the jumping numbers of $\mathfrak{a}$ supported at $\mu$ :

$$
\mathcal{H}_{\mu}^{\mathfrak{a}}=\left\{\frac{t}{d_{\mu}} \mid t+\left(v_{\Gamma}(\mu)-2\right) V_{\mu, \mu}-\sum_{\nu \sim \mu} s_{\nu}^{\mu}\left\lceil t \sum_{i \in \Gamma_{\nu}^{\mu}} \frac{\widehat{d}_{i} V_{\mu, i}}{s_{\nu}^{\mu} d_{\mu}}\right]^{+} \in S^{\mu}\right\},
$$

where \lceil\rceil$^{+}$means rounding up to the nearest positive integer. Note that a jumping number is always supported at a vertex which is either a star or corresponds to a simple factor of the ideal (see Lemma 9). 
In the proof we look at the factors of divisors. Given a vertex $\mu$ we introduce two transforms of divisors by means of which it is possible 'bring' factors from each branch emanating from $\mu$ to the closest vertex adjacent to $\mu$ and 'distribute' a part of a factor from $\mu$ to the adjacent vertices. Suppose that $\xi=\xi_{F}$ is supported at a $\mu$. Using these transformations we can modify either $F$ or $D$ or both in such a way that we still have $\xi=\xi_{F}$. In particular, we can assume that the divisor $D$ has factors only at the vertices adjacent to $\mu$. In this process the properties of the mappings $\rho_{[\mu, \gamma]}: \Gamma \rightarrow \mathbb{Q}$, where $\mu$ and $\gamma$ are fixed vertices, and

$$
\nu \mapsto \frac{V_{\gamma, \nu}}{V_{\mu, \nu}}
$$

play a crucial role. In particular, we prove in Lemma 10 that $\rho_{[\mu, \gamma]}$ is strictly increasing along the path going from $\mu$ to $\gamma$, and stays constant on any path going away from this path. Finally, we show in Example 31 how our formula works in practice.

\section{PRELIMINARIES}

In this paper, we make use of the Zariski-Lipman theory of complete ideals. The general setting here is similar to that discussed in our paper [6]. For the reader's convenience, we collect here some basic concepts and notation. More details can be found in [10], [2], [11] and [8].

About Zariski-Lipman theory. Let $\mathfrak{a}$ be a complete ideal of finite colength in a two-dimensional regular local ring $R$ having an algebraically closed residue field. Let $\pi: X \rightarrow \operatorname{Spec}(R)$ be a principalization of $\mathfrak{a}$. Then $X$ is a regular scheme and $\mathfrak{a} \mathcal{O}_{X}=\mathcal{O}_{X}(-D)$ for an effective Cartier divisor $D$. The morphism $\pi$ is a composition of point blowups of regular schemes

$$
\pi: X=X_{N+1} \stackrel{\pi_{N}}{\longrightarrow} \cdots \stackrel{\pi_{2}}{\longrightarrow} X_{2} \stackrel{\pi_{1}}{\longrightarrow} X_{1}=\operatorname{Spec} R,
$$

where $\pi_{\mu}$ is the blowup of $X_{\mu}$ at a closed point $x_{\mu} \in X_{\mu}$. Let $E_{\mu}$ be the strict and $E_{\mu}^{*}$ the total transform of the exceptional divisor $\pi_{\mu}^{-1}\left\{x_{\mu}\right\}$ on $X$. We write $v_{\mu}$ for the discrete valuation associated to the discrete valuation ring $\mathcal{O}_{X, E_{\mu}}$, so that $v_{\mu}$ is the $\mathfrak{m}_{X_{\mu}, x_{\mu}}$-adic order valuation.

A point $x_{\mu}$ is infinitely near to a point $x_{\nu}$, if the projection $X_{\mu} \rightarrow X_{\nu}$ maps $x_{\mu}$ to $x_{\nu}$. Further, $x_{\mu}$ is proximate to $x_{\nu}$, denoted by $\mu \succ \nu$, if $x_{\mu}$ lies on the strict transform of $\pi_{\nu}^{-1}\left\{x_{\nu}\right\}$ on $X_{\mu}$. Note that a point can be proximate to at most two points. The proximity matrix is

$$
P:=\left(p_{\mu, \nu}\right)_{N \times N}, \text { where } p_{\mu, \nu}=\left\{\begin{aligned}
1, & \text { if } \mu=\nu \\
-1, & \text { if } \mu \succ \nu \\
0, & \text { otherwise. }
\end{aligned}\right.
$$

We write $Q=\left(q_{\mu, \nu}\right)_{N \times N}:=P^{-1}$, so that $P Q=1$.

Besides the obvious one, the lattice $\Lambda:=\mathbb{Z} E_{1} \oplus \ldots \oplus \mathbb{Z} E_{N}$ of exceptional divisors on $X$ has two other convenient bases, namely $\left\{E_{1}^{*}, \ldots, E_{N}^{*}\right\}$ and $\left\{\widehat{E}_{1}, \ldots, \widehat{E}_{N}\right\}$, where $E_{\mu} \cdot \widehat{E}_{\nu}=E_{\mu}^{*} \cdot E_{\nu}^{*}=-\delta_{\mu, \nu}$. Throughout this paper we use 
the practice that if an upper case letter, say $G$, denotes a divisor $G \in \Lambda$, then the corresponding lower case letter possibly with an accent mark denotes the coefficient vector with respect to the appropriate base. In particular, writing

$$
G=g_{1} E_{1}+\ldots+g_{N} E_{N}=g_{1}^{*} E_{1}^{*}+\ldots+g_{N}^{*} E_{N}^{*}=\widehat{g}_{1} \widehat{E}_{1}+\ldots+\widehat{g}_{N} \widehat{E}_{N}
$$

with $g=\left(g_{\nu}\right), g^{*}=\left(g_{\nu}^{*}\right)$ and $\widehat{g}=\left(\widehat{g}_{\nu}\right)$, we get the following base change formulas:

$$
g^{*}=g P^{\mathrm{T}} \text { and } \widehat{g}=g P^{\mathrm{T}} P=g^{*} P .
$$

In many cases, we regard $\Lambda$ as a subset of $\Lambda_{\mathbb{Q}}:=\mathbb{Q} \otimes \Lambda$. We call the vector $\widehat{g}$ the factorization vector and $g$ the valuation vector of the divisor. Note that $g=\widehat{g} V$, where $V:=\left(P^{\mathrm{T}} P\right)^{-1}$ is called the valuation matrix. Set

$$
w_{\Gamma}(\mu):=-E_{\mu}^{2}=1+\#\{\nu \mid \nu \succ \mu\} .
$$

We then get the formulas

$$
\widehat{g}_{\mu}=g_{\mu}^{*}-\sum_{\nu \succ \mu} g_{\nu}^{*}=w_{\Gamma}(\mu) g_{\mu}-\sum_{\nu \sim \mu} g_{\nu} \quad(\mu=1, \ldots, N) .
$$

Especially, this yields

$$
w_{\Gamma}(\eta) V_{\mu, \eta}=\sum_{i \sim \eta} V_{\mu, i}+\delta_{\mu, \eta}
$$

Recall that a divisor $F \in \Lambda$ is antinef if $\widehat{f}_{\nu}=-F \cdot E_{\nu} \geq 0$ for all $\nu=$ $1, \ldots, N$. Equivalently, the proximity inequalities

$$
f_{\mu}^{*} \geq \sum_{\nu \succ \mu} f_{\nu}^{*} \quad(\mu=1, \ldots, N)
$$

hold. Note that they can also be expressed in the form

$$
w_{\Gamma}(\mu) f_{\mu} \geq \sum_{\nu \sim \mu} f_{\nu} \quad(\mu=1, \ldots, N) .
$$

In fact, if $F \neq 0$ is antinef, then also $f_{\nu}>0$ for all $\nu=1, \ldots, N$. There is a one to one correspondence between the antinef divisors in $\Lambda$ and the complete ideals of finite colength in $R$ generating invertible $\mathcal{O}_{X}$-sheaves, given by $F \leftrightarrow \Gamma\left(X, \mathcal{O}_{X}(-F)\right)$.

An ideal is called simple if it cannot be expressed as a product of two proper ideals. By the famous result of Zariski, every complete ideal factorizes uniquely into a product of simple complete ideals. More precisely, we can present a complete ideal $\mathfrak{a}$ as a product

$$
\mathfrak{a}=\mathfrak{p}_{1}^{\widehat{d}_{1}} \cdots \mathfrak{p}_{N}^{\widehat{d}_{N}}
$$

where $\mathfrak{p}_{\mu} \subset R$ denotes the simple complete ideal of finite colength corresponding to the exceptional divisor $\widehat{E}_{\mu}$ and $\widehat{d}_{i} \in \mathbb{N}$ for every $i$. By (1)

$$
\widehat{E}_{\mu}=\sum_{\nu} q_{\mu, \nu} E_{\nu}^{*}=\sum_{\nu, \rho} q_{\nu, \rho} q_{\mu, \rho} E_{\nu} .
$$


In particular, we observe the reciprocity formula

$$
v_{\nu}\left(\mathfrak{p}_{\mu}\right)=\sum_{\rho} q_{\nu, \rho} q_{\mu, \rho}=v_{\mu}\left(\mathfrak{p}_{\nu}\right) \quad(\mu, \nu=1, \ldots, N),
$$

in short, $V=V^{\mathrm{T}}$. Recall that the canonical divisor is $K=\sum_{\nu} E_{\nu}^{*}$. If $k=\left(k_{\nu}\right)$ and $\widehat{k}=\left(\widehat{k}_{\nu}\right)$ are the appropriate coefficient vectors, we have

$$
k E=\widehat{k} \widehat{E}=K .
$$

The formulas (1) yield

$$
k_{\nu}=\sum_{\mu} q_{\nu, \mu} \quad \text { and } \quad \widehat{k}_{\nu}=E_{\nu}^{2}+2 \quad(\nu=1, \ldots, N) .
$$

Dual graph. The dual graph $\Gamma$ associated to our principalization is a tree, where the vertices correspond one to one to the exceptional divisors and an edge between two adjacent vertices, $\gamma \sim \eta$, means that the corresponding exceptional divisors $E_{\gamma}$ and $E_{\eta}$ intersect. A vertex $\gamma$ corresponding to the exceptional divisor $E_{\gamma}$ is weighted by the number $w_{\Gamma}(\gamma)$. We say that a vertex $\gamma$ is proximate to another vertex $\eta$ if $p_{\gamma, \eta}=-1$. It is free if it is proximate to at most one vertex. We may also say that $\gamma$ is infinitely near to $\eta$, and write $\eta \subset \gamma$, if this is the case with the corresponding points. The root of $\Gamma$ is the vertex $\tau_{0}$ for which $\tau_{0} \subset \gamma$ for every $\gamma \in \Gamma$.

Blowing up a point on $E_{\gamma}$ expands the dual graph by adding a vertex $\nu$ corresponding to the exceptional divisor of the blowup. The weight of the new vertex is one and the weights of the adjacent vertices are increased by one. In [14, Definition 5.1] such expansions are called elementary modifications. There are two kinds of elementary modifications. If $E_{\gamma}$ is the only exceptional divisor containing the center of blowup so that $\gamma \sim \nu$ forms the only new edge, then the elementary modification is of the first kind:

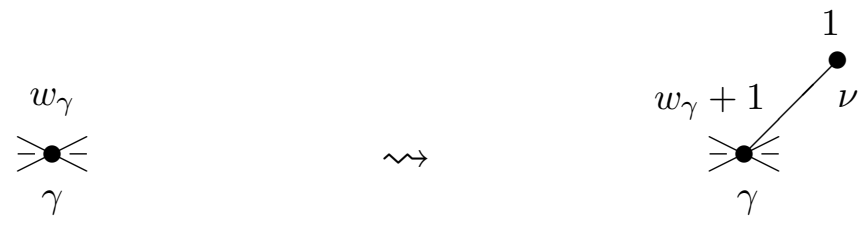

If the center of blowup is the intersection point of $E_{\gamma}$ and another exceptional divisor, say $E_{\eta}$, then the edge $\gamma \sim \eta$ is replaced by the edges $\gamma \sim \nu$ and $\nu \sim \eta$, and the elementary modification is of the second kind:

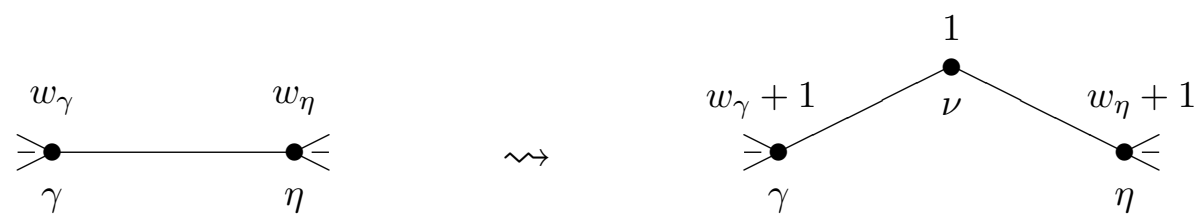

Let us write

$$
\Gamma(\nu, U) \text { where } \underset{5}{=}=\{\gamma \in \Gamma \mid \gamma \prec \nu\}
$$


for an elementary modification of the graph $\Gamma$ by adding a vertex $\nu$ adjacent to vertices $\gamma \in U$. Note that $U$ consists of at most two vertices. Note also that if the graph is empty then the elementary modification is defined to be of the first kind containing only the root vertex. Following [14, Definition 5.2], a dual graph dominates a dual graph $\Gamma$, if it can be obtained from $\Gamma$ by a sequence of elementary modifications. Obviously, a sequence of point blowups correspond to a sequence of elementary modifications. Especially, the dual graph of our principalization can be obtained from the graph containing only the root vertex through successive elementary modifications (c.f. [14, Remark $5.5])$.

In a way, the matrix $P^{\mathrm{T}} P$ represents the dual graph because the diagonal elements $\left(P^{\mathrm{T}} P\right)_{\nu, \nu}=-E_{\nu}^{2}$ correspond with the weights of the vertices while outside the diagonal the element $\left(P^{\mathrm{T}} P\right)_{\mu, \nu}=-E_{\mu} \cdot E_{\nu}$ is -1 if $E_{\mu}$ and $E_{\nu}$ intersect and otherwise zero.

The valence $v_{\Gamma}(\mu)$ of a vertex $\mu$ means the number of vertices adjacent to it. If $v_{\Gamma}(\mu) \geq 3$, then $\mu$ is called a star. If $v_{\Gamma}(\mu) \leq 1$, then we call it an end. The vertices adjacent to $\mu$ correspond one to one to the branches emanating from $\mu$, which can be defined as follows:

Definition 1. For any two vertices $\mu$ and $\nu$ in $\Gamma$, let $\Gamma_{\nu}^{\mu}$ denote the maximal connected subgraph of $\Gamma$ containing $\nu$ but not $\mu\left(\Gamma_{\mu}^{\mu}=\emptyset\right)$. We say $\Gamma_{\nu}^{\mu}$ is a branch emanating from $\mu$ towards $\nu$. A branch $\Gamma_{\nu}^{\mu}$ is anterior to $\mu$, if $\mu$ is infinitely near to some of its vertices. Otherwise we say it is posterior to $\mu$.

Observe that every branch emanating from $\mu$ is either anterior or posterior to $\mu$, and for those we immediately get the following result:

Proposition 1. The unempty posterior branches of $\mu$ correspond one to one to the free vertices, which are proximate to $\mu$, whereas the anterior branches are in one to one correspondence with the vertices to which $\mu$ is proximate to.

Proof. The claim is trivial if $\mu$ is the only vertex, meaning that there are no unempty branches. We shall proceed by induction on the number of vertices. Suppose $\Gamma=\Gamma^{\prime}(\eta, U)$ and the claim holds for $\Gamma^{\prime}$. Observe that for any $\mu \in \Gamma$, $\mu$ is not proximate to $\eta$.

If $\mu=\eta$, then $\gamma \prec \mu$ exactly when $\gamma \sim \mu$, so that $\mu$ is infinitely near to any adjacent vertex. Obviously, the branches $\Gamma_{\gamma}^{\mu}$ correspond one to one to the vertices $\gamma \sim \mu$. Thus the claim is clear in this case.

Suppose that $\mu \neq \eta$. If $\eta$ is not a free vertex proximate to $\mu$, then the blowup just augments an existing branch of $\Gamma^{\prime}$, i. e., the branches of $\Gamma$ emanating from $\mu$ correspond one to one to those of $\Gamma^{\prime}$. Because the proximity relations are preserved under blowup, the claim follows. If $\eta$ is a free blowup of $\mu$, then $\mu \prec \eta$ and $\Gamma_{\eta}^{\mu}=\{\eta\}$ forms a new branch, which corresponds to the vertex $\eta$. For the rest of the branches emanating from $\mu$ the correspondence is inherited from $\Gamma^{\prime}$. 
Recall that a vertex is proximate to at most two vertices. Subsequently, there are at most two branches anterior to $\mu \in \Gamma$ depending on whether $\mu$ is free or not.

The distance between two vertices $\mu, \nu \in \Gamma$ is defined as the length of the path $[\nu, \mu]$, i. e.,

$$
d(\nu, \mu):=\min \left\{r \mid \nu=\nu_{0} \sim \cdots \sim \nu_{r}=\mu, \text { where } \nu_{0}, \ldots, \nu_{r} \in \Gamma\right\},
$$

Furthermore, if $T \subset \Gamma$, we set

$$
d(\nu, T):=\min \{d(\nu, \mu) \mid \mu \in T\} .
$$

If $d(\nu, T)=1$, then we write $\nu \sim T$.

Definition 2. A pair $(\gamma, \tau)$ is associated to $\mu$, if $\gamma$ and $\tau$ satisfy the following three conditions:

i) $\gamma \subset \tau \subset \mu$, i. e., $\mu$ is infinitely near to $\tau$ which is infinitely near to $\gamma$;

ii) $\tau$ is free and infinitely near to every free vertex $\nu \subset \mu$;

iii) $\gamma$ is not free and infinitely near to every non free vertex $\nu \subset \tau$, unless every $\nu \subset \tau$ is free in which case $\gamma=\tau_{0}$ is the root.

The sequence of pairs $\left(\left(\gamma_{i}, \tau_{i+1}\right)\right)_{i=0}^{g}$ is associated to $\mu:=\gamma_{g+1}$, if it holds for $i=0, \ldots, g$ that $\left(\gamma_{i}, \tau_{i+1}\right)$ is the pair associated to $\gamma_{i+1}$.

Remark 2. Let $\Gamma$ be the dual graph of $\mu$, i. e., the simple dual graph which consists of all the vertices to which $\mu$ is infinitely near to. Observe that we may always reach this situation by repeatedly blowing down any vertex different from $\mu$ having a weight one. If $\left(\left(\gamma_{i}, \tau_{i+1}\right)\right)_{i=0}^{g}$ is now the sequence associated to $\mu$, then $\gamma_{0}=\tau_{0}$ is the root, $\tau_{0}, \ldots, \tau_{g+1}$ are exactly the end vertices of $\Gamma$ while $\gamma_{1}, \ldots, \gamma_{g}$ are its stars (cf. [8, Proposition 4.3]). Note that the integer $g$, i. e., the number of star vertices of the dual graph, is denoted by $g^{*}$ in [8, Notation $3.3]$.

Remark 3. As the relation $\nu \subset \mu$ induces a partial order on $\Gamma$, we might give the definition as follows: a pair $(\gamma, \tau)$ is associated to $\mu$, if $\tau$ is maximal among the free points to which $\mu$ is infinitely near to, and $\gamma$ is maximal among the non free points to which $\tau$ is infinitely near to. The graph below illustrates an example of a sequence of pairs associated to a vertex.

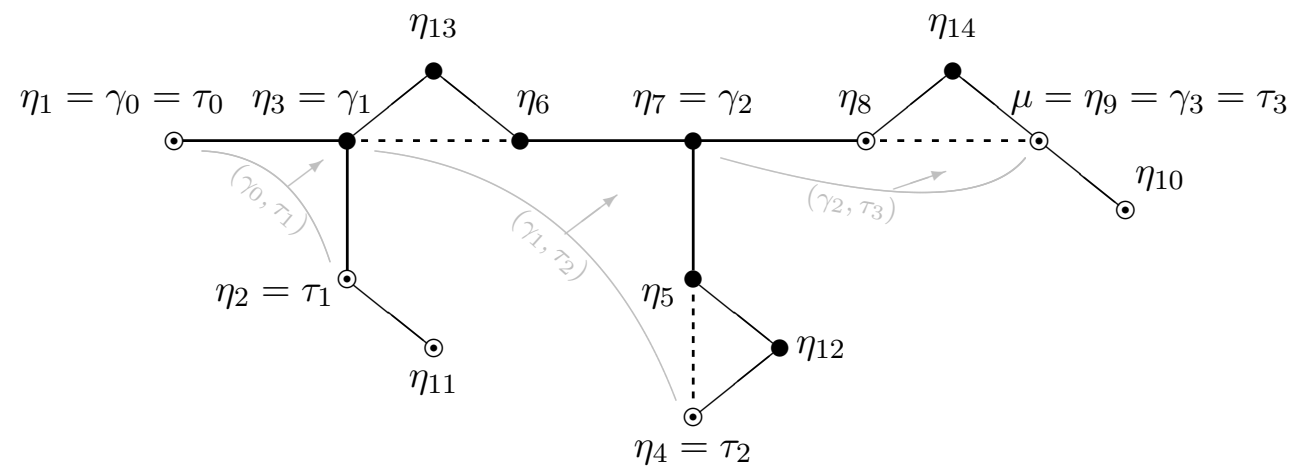


Here the open circles represent free points. We now have $\eta_{1} \subset \cdots \subset \eta_{10}$. Moreover, $\eta_{2} \subset \eta_{11}, \eta_{5} \subset \eta_{12}, \eta_{6} \subset \eta_{13}$ and $\eta_{9} \subset \eta_{14}$. Since we are interested in the vertices to which $\mu$ is infinitely near to, we may concentrate on the chain $\eta_{1} \subset \cdots \subset \eta_{9}$ or, in the dual graph, blow down the vertices $\eta_{i}$ with $i>9$. The dashed lines in the graph represent the edges emerging when blowing down. Obviously, the maximal free point to which $\mu=\eta_{9}$ is infinitely near to is $\mu$ itself, and further, the maximal non free point to which $\mu$ is infinitely near to is $\eta_{7}$. Thus the pair $\left(\eta_{7}, \mu\right)$ is associated to $\mu$. Similarly, the pair $\left(\eta_{3}, \eta_{4}\right)$ is associated to $\eta_{7}$ and $\left(\eta_{1}, \eta_{2}\right)$ is associated to $\eta_{3}$.

Jumping numbers. We will next recall the definition of jumping numbers. A general reference for jumping numbers is the fundamental article [3]. For a nonnegative rational number $\xi$, the multiplier ideal $\mathcal{J}\left(\mathfrak{a}^{\xi}\right)$ is defined to be the ideal

$$
\mathcal{J}\left(\mathfrak{a}^{\xi}\right):=\Gamma\left(X, \mathcal{O}_{X}(K-\lfloor\xi D\rfloor)\right) \subset R,
$$

where $D=d_{1} E_{1}+\cdots+d_{N} E_{N}$ is the divisor corresponding to $\mathfrak{a}$ and $\lfloor\xi D\rfloor$ denotes the integer part of $\xi D$. It is now known that there is an increasing discrete sequence

$$
0=\xi_{0}<\xi_{1}<\xi_{2}<\cdots
$$

of rational numbers $\xi_{i}$ characterized by the properties that $\mathcal{J}\left(\mathfrak{a}^{\xi}\right)=\mathcal{J}\left(\mathfrak{a}^{\xi_{i}}\right)$ for $\xi \in\left[\xi_{i}, \xi_{i+1}\right)$, while $\mathcal{J}\left(\mathfrak{a}^{\xi_{i+1}}\right) \subsetneq \mathcal{J}\left(\mathfrak{a}^{\xi_{i}}\right)$ for every $i$. The numbers $\xi_{1}, \xi_{2}, \ldots$, are called the jumping numbers of $\mathfrak{a}$. The following Proposition 4, which is fundamental for the rest of this article, results from [8, Proposition 6.7 and Proposition 7.2].

Proposition 4. Let $\mathfrak{a} \subset R$ be a complete ideal of finite colength. Then $\xi$ is a jumping number of $\mathfrak{a}$ if and only if there exists an antinef divisor $F=f E \in \Lambda$ such that

$$
\xi=\xi_{F}:=\min _{\nu} \frac{f_{\nu}+k_{\nu}+1}{d_{\nu}} .
$$

Moreover, if $\mathfrak{b}$ is the complete ideal corresponding to $F$, then

$$
\xi=\inf \left\{c \in \mathbb{Q}_{>0} \mid \mathcal{J}\left(\mathfrak{a}^{c}\right) \nsupseteq \mathfrak{b}\right\} .
$$

Notation. We write for any two divisors $F=f E, G=g E \in \Lambda_{\mathbb{Q}}$ and for any vertex $\nu$

For any integer $a$ we set

$$
\lambda(F, G ; \nu):=\frac{f_{\nu}+k_{\nu}+1}{g_{\nu}} .
$$

$$
\lambda(a, \nu)=\lambda(a, D ; \nu):=\lambda(a E, D ; \nu) .
$$

Furthermore, we call the set

$$
\left\{\nu \in \Gamma \mid \lambda\left(f_{\nu}, \nu\right)=\xi\right\}
$$

the support of the jumping number $\xi$ with respect to the divisor $F$. The set of jumping numbers of $\mathfrak{a}$ supported at a vertex $\mu \in \Gamma$ is denoted by

$$
\mathcal{H}_{\mu}^{\mathfrak{a}}:=\left\{\xi_{F} \mid F \in \Lambda \text { is antinef and } \xi_{F}=\lambda(F, D ; \mu)\right\} .
$$


Recall that the function $\lambda_{F}:|\Gamma| \rightarrow \mathbb{Q}$, where $F=\sum_{\nu \in \Gamma} f_{\nu} E_{\nu}$ is a divisor and $\lambda_{F}(\nu)=\lambda\left(f_{\nu}, \nu\right)$, makes the dual graph as an ordered tree. In [6] we investigated this kind of ordered tree structures, and further, we proved that a number being a jumping number is equivalent to the existence of certain kind of ordered tree structures. In the sequel, we make use of these results.

Remark 5. Note that in [6] and [8] $\Gamma$ is the dual graph of the minimal principalization of $\mathfrak{a}$. We may loosen this restriction and consider the dual graph of a principalization of the ideal. In the sequel, we may think $\Gamma$ as a dual graph of any ideal corresponding to some antinef divisor in $\Lambda$. This is convenient, and it is possible because if $\mathfrak{b}$ is such an ideal, then the principalization corresponding to $\Gamma$ is a principalization of $\mathfrak{b}$, and the minimal principalization is obtained by blowing down. Observe that the ordered tree structures behave accordingly. Suppose that the divisor corresponding to $\mathfrak{b}$ is $g E$ and that the dual graph $\Gamma_{\mathfrak{b}}$ of its minimal principalization is obtained by blowing down a vertex $\nu \in \Gamma$, then the valuation matrix of $\mathfrak{b}$ is just a restriction of that of $\mathfrak{a}$. For a divisor $f E \in \Lambda_{\mathbb{Q}}$ and for a vertex $\gamma \in \Gamma_{\mathfrak{b}}$ we get $\lambda\left(\left.f E\right|_{\Gamma_{\mathfrak{b}}},\left.g E\right|_{\Gamma_{\mathfrak{b}}} ; \gamma\right)=\lambda(f E, g E ; \gamma)$. Thus the ordered tree structures provided by $\lambda$ (see [6]) can be obtained as restrictions, as well.

Recall our main result in [6, Theorem 1]:

Theorem 6 (Theorem 1 in [6]). We have $\xi \in \mathcal{H}_{\mu}^{\mathfrak{a}}$ if and only if there is a (connected) set $U \subset \Gamma$ containing $\mu$ and a set of nonnegative integers

$$
\left\{a_{\eta} \in \mathbb{N} \mid d(\eta, U) \leq 1\right\}
$$

satisfying

i) $\lambda\left(a_{\eta}, \eta\right)>\xi=\lambda\left(a_{\gamma}, \gamma\right)$ for every $\gamma \in U$ and $\eta \sim U$;

ii) $w_{\Gamma}(\gamma) a_{\gamma} \geq \sum_{\nu \sim \gamma} a_{\nu}$ for every $\gamma \in U$.

For further use, we also give here a refined versions of [6, Lemma 5] and [6, Lemma 7].

Lemma 7. Given any vertex $\gamma \in \Gamma$ and any nonnegative integer $a_{\gamma}$, we may choose for every vertex $\eta \sim \gamma$ a nonnegative integer $a_{\eta}$ so that

$$
w_{\Gamma}(\gamma) a_{\gamma} \geq \sum_{\eta \sim \gamma} a_{\eta} \text { and } \lambda\left(a_{\eta}, \eta\right) \geq \lambda\left(a_{\gamma}, \gamma\right)
$$

where the latter inequality holds for each $\eta \sim \gamma$ except at most one. More precisely, if

$$
\{\eta \mid \eta \sim \gamma\}=\left\{\eta_{1}, \ldots \eta_{m}\right\}
$$

where $m>1$, then the following is true:

1) If it is possible to find a nonnegative integer $a_{\eta_{1}}$ with $\lambda\left(a_{\eta_{1}}, \eta_{1}\right)=$ $\lambda\left(a_{\gamma}, \gamma\right)$, then one may choose the other integers $a_{\eta_{j}}$ so that

$$
\lambda\left(a_{\eta_{2}}, \eta_{2}\right) \geq \lambda\left(a_{\gamma}, \gamma\right) \text { and } \lambda\left(a_{\eta_{j}}, \eta_{j}\right)>\lambda\left(a_{\gamma}, \gamma\right)
$$

for all $2<j \leq m$. 
2) If it is possible to find a nonnegative integer $a_{\eta_{1}}$ satisfying $\lambda\left(a_{\eta_{1}}, \eta_{1}\right)<$ $\lambda\left(a_{\gamma}, \gamma\right)$ or, in the case $\widehat{d}_{\gamma}>0, \lambda\left(a_{\eta_{1}}, \eta_{1}\right)=\lambda\left(a_{\gamma}, \gamma\right)$, then one can choose the other integers $a_{\eta}$ in such a way that

$$
\lambda\left(a_{\eta_{j}}, \eta_{j}\right)>\lambda\left(a_{\gamma}, \gamma\right)
$$

holds for every $1<j \leq m$.

Proof. The proof is conducted in [6, Lemma 5] except for the amendment in 1 ), which claims that if we have nonnegative integers $a_{\eta_{1}}$ and $a_{\eta_{2}}$ satisfying

$$
\lambda\left(a_{\eta_{2}}, \eta_{2}\right)>\lambda\left(a_{\gamma}, \gamma\right)=\lambda\left(a_{\eta_{1}}, \eta_{1}\right)>\lambda\left(a_{\eta_{2}}-1, \eta_{2}\right),
$$

then we may find nonnegative integers $a_{\eta_{j}}$ for $2<j \leq m$ so that

$$
w_{\Gamma}(\gamma) a_{\gamma}=\sum_{j=1}^{m} a_{\eta_{j}} \text { and } \lambda\left(a_{\eta_{j}}, \eta_{j}\right) \geq \lambda\left(a_{\gamma}, \gamma\right),
$$

where the inequality is strict for $1<j \leq m$.

To prove that, suppose that Equation (5) holds. For $\mu, \nu \in \Gamma$, write

$$
\alpha_{\mu, \nu}:=k_{\nu}+1-\frac{d_{\nu}}{d_{\mu}}\left(k_{\mu}+1\right) .
$$

Note that if $a_{\eta_{2}}=0$, then by [6, Lemma 3 a)]

$$
a_{\gamma}-1 \leq a_{\gamma}+\alpha_{\eta_{2}, \gamma}=d_{\gamma}\left(\lambda\left(a_{\gamma}, \gamma\right)-\lambda\left(a_{\eta_{2}}, \eta_{2}\right)\right),
$$

which must be negative. Therefore $a_{\gamma}=0$, and then similarly, by [6, Lemma 3 a)],

$$
a_{\eta_{1}}-1<a_{\eta_{1}}+\alpha_{\gamma, \eta_{1}}=d_{\eta_{1}}\left(\lambda\left(a_{\eta_{1}}, \eta_{1}\right)-\lambda\left(a_{\gamma}, \gamma\right)\right)=0
$$

so that $a_{\eta_{1}}=0$, but then the claim follows from [6. Lemma $\left.3 \mathrm{~b}\right)$ ].

Assume then that $a_{\eta_{2}}>0$ and set $a_{\eta_{2}}^{\prime}:=a_{\eta_{2}}-1$. Now $\lambda\left(a_{\eta_{2}}^{\prime}, \eta_{2}\right)<\lambda\left(a_{\gamma}, \gamma\right)$, and by [6, Lemma 5] we may find nonnegative integers $a_{\eta_{j}}^{\prime}$ for $1 \leq j \leq m$, $j \neq 2$, so that

$$
w_{\Gamma}(\gamma) a_{\gamma}=\sum_{j=1}^{m} a_{\eta_{j}}^{\prime} \text { and } \lambda\left(a_{\eta_{j}}^{\prime}, \eta_{j}\right)>\lambda\left(a_{\gamma}, \gamma\right) \text { for } j \neq 2 .
$$

But then $\lambda\left(a_{\eta_{1}}^{\prime}, \eta_{1}\right)>\lambda\left(a_{\eta_{1}}, \eta_{1}\right)$. Clearly, we may choose the integers $a_{\eta_{j}}^{\prime}$ so that $a_{\eta_{1}}^{\prime}=a_{\eta_{1}}+1$. It follows that

$$
w_{\Gamma}(\gamma) a_{\gamma}=a_{\eta_{1}}+a_{\eta_{2}}+\sum_{j=3}^{m} a_{\eta_{j}}^{\prime} \text { and } \lambda\left(a_{\eta_{j}}^{\prime}, \eta_{j}\right)>\lambda\left(a_{\gamma}, \gamma\right) \text { for } 2<j \leq m \text {. }
$$

Choosing now $a_{\eta_{j}}=a_{\eta_{j}}^{\prime}$ for $2<j \leq m$ yields the claim.

Practically, the lemma shows that if $\lambda_{F}(\mu)$ is a local minimum for a function $\lambda_{F}$ where $F$ is an effective divisor, then we may find an antinef divisor $A=$ $\sum_{\nu} a_{\nu} E_{\nu}$ for which $\lambda\left(a_{\mu}, \mu\right)=\lambda\left(f_{\mu}, \mu\right)$ is the global minimum of the function $\lambda_{A}$. The only problem that may arise in finding such integers $a_{\nu}$ is the situation where we already have integers $a_{\gamma}$ and $a_{\tau}$ with $\lambda\left(a_{\gamma}, \gamma\right)=\lambda\left(a_{\tau}, \tau\right)$, and $\tau$ is an end vertex. We cannot go on choosing integers $a_{\nu}$ with $\tau \sim \nu \neq \gamma$ and 
$\lambda\left(a_{\nu}, \nu\right)=\lambda\left(a_{\tau}, \tau\right)$, because there are no such vertices, and it may happen that $\widehat{a}_{\tau}:=w_{\gamma}(\tau) a_{\tau}-a_{\gamma}<0$. This is the reason why we cannot apply 1) of Lemma 7 to an end. Nevertheless, rephrasing [6, Lemma 7], the next Lemma shows that 2) of Lemma 7 is applicable even if the vertex in question is an end.

Lemma 8. Suppose $\tau$ is an end and $\gamma$ is adjacent to it. If $a_{\tau}$ and $a_{\gamma}$ are such integers that $\lambda\left(a_{\gamma}, \gamma\right) \leq \lambda\left(a_{\tau}, \tau\right)$, where the equality holds only if $\widehat{d}_{\tau}>0$, then

$$
w_{\Gamma}(\tau) a_{\tau} \geq a_{\gamma} .
$$

Proof. By Equation (4) we know that $\widehat{k}_{\tau}=2-w_{\Gamma}(\tau)$. On the other hand, by Equation (2) we have $\widehat{k}_{\tau}=w_{\Gamma}(\tau) k_{\tau}-k_{\gamma}$. Thus $w_{\Gamma}(\tau)\left(k_{\tau}+1\right)=k_{\gamma}+2$. Moreover, by Equation (2) we get $w_{\Gamma}(\tau) d_{\tau}=d_{\gamma}+\widehat{d}_{\tau}$. This shows that

$$
\lambda\left(a_{\tau}, \tau\right)=\frac{w_{\Gamma}(\tau)\left(a_{\tau}+k_{\tau}+1\right)}{w_{\Gamma}(\tau) d_{\tau}}=\frac{w_{\Gamma}(\tau) a_{\tau}+k_{\gamma}+2}{d_{\gamma}+\widehat{d}_{\tau}}
$$

Therefore we see that

$$
\lambda\left(a_{\gamma}, \gamma\right)=\frac{a_{\gamma}+k_{\gamma}+1}{d_{\gamma}}<\frac{\left(w_{\Gamma}(\tau) a_{\tau}+1\right)+k_{\gamma}+1}{d_{\gamma}},
$$

which implies that $a_{\gamma}<w_{\Gamma}(\tau) a_{\tau}+1$, as wanted.

By using these results we may construct suitable ordered tree structures, which in turn can prove that certain rationals are jumping numbers for our ideal supported at the desired vertex or vertices. The next lemma shows that in order to determine the jumping numbers of an ideal, we just need to know the jumping numbers supported at a vertex which is either a star or corresponds to a simple factor of the ideal.

Lemma 9. A support of a jumping number contains a vertex which is either a star or corresponds to a simple factor of the ideal.

Proof. Let $\Gamma$ be a dual graph of an ideal $\mathfrak{a}=\prod_{\nu \in \Gamma} \mathfrak{p}_{\nu}^{\widehat{d}_{\nu}}$. Suppose $\xi$ is a jumping number of $\mathfrak{a}$ supported at a vertex $\gamma \in \Gamma$, for which $\widehat{d}_{\gamma}=0$ and $v_{\Gamma}(\gamma)<3$. By Proposition 4 we have an antinef divisor $F$ for which $\xi=\xi_{F}$. Further, we have $\xi=\lambda\left(f_{\gamma}, \gamma\right) \leq \lambda\left(f_{\nu}, \nu\right)$ for any $\nu \in \Gamma$. As $\widehat{k}_{\gamma}=2-w_{\Gamma}(\gamma)$ by (4), and on the other hand, $\widehat{k}_{\gamma}=w_{\Gamma}(\gamma) k_{\gamma}-\sum_{\eta \sim \gamma} k_{\eta}$, we see that $w_{\Gamma}(\gamma)\left(k_{\gamma}+1\right)=2+\sum_{\eta \sim \gamma} k_{\eta}$. By this and by (2) we obtain

$$
\lambda\left(f_{\gamma}, \gamma\right)=\frac{w_{\Gamma}(\gamma) f_{\gamma}+w_{\Gamma}(\gamma)\left(k_{\gamma}+1\right)}{w_{\Gamma}(\gamma) d_{\gamma}}=\frac{\widehat{f}_{\gamma}+2-v_{\Gamma}(\gamma)+\sum_{\eta \sim \gamma}\left(f_{\eta}+k_{\eta}+1\right)}{\widehat{d}_{\gamma}+\sum_{\eta \sim \gamma} d_{\eta}}
$$

Since $\widehat{d}_{\gamma}=0, v_{\Gamma}(\gamma)<3$ and $\lambda\left(f_{\eta}, \eta\right) \geq \lambda\left(f_{\gamma}, \gamma\right)$, the above yields $v_{\Gamma}(\gamma)=2$, $\widehat{f}_{\gamma}=0$ and $\lambda\left(f_{\eta}, \eta\right)=\xi$ for any $\eta \sim \gamma$. In other words, $\gamma$ has exactly two adjacent vertices, which both are in the support of $\xi$. If neither of them is a star nor corresponds to a factor, we may apply the above to them. Because the dual graph contains finitely many vertices, we must eventually come up with a vertex in the support of $\xi$, which is either a star or corresponds to a factor. 


\section{ModificATiONS OF THE FACTORIZATION}

Let $\mathfrak{a}, D$ and $\Gamma$ be as above and let $V$ be the valuation matrix, $\widehat{d} \in \mathbb{Q}^{\Gamma}$ the factorization vector and $d=\widehat{d} V$ the valuation vector of $\mathfrak{a}$. According to 6 , Theorem 1 and Lemma 6], we may find for any antinef divisor $F=f E \in \Lambda$ an antinef divisor $G=g E$ such that $\widehat{g}_{\nu}>0$ only if $\nu$ is an end of $\Gamma$ and $\xi_{F}=\xi_{G}$. In this paper we further investigate divisors corresponding to a jumping number and develop a method to modify them in order to find ideals sharing the jumping numbers supported at a given vertex. To begin with, let us consider the mapping $\rho_{[\mu, \gamma]}: \Gamma \rightarrow \mathbb{Q}$, where

$$
\rho_{[\mu, \gamma]}: \nu \mapsto \frac{V_{\gamma, \nu}}{V_{\mu, \nu}}
$$

Lemma 10. The mapping $\rho_{[\mu, \gamma]}$ is strictly increasing on the path going from $\mu$ to $\gamma$ and it stays constant on any path going away from $[\mu, \gamma]$, in other words, $\rho_{[\mu, \gamma]}\left(\nu_{1}\right)<\rho_{[\mu, \gamma]}\left(\nu_{2}\right)$ if and only if $\left[\mu, \nu_{1}\right] \cap[\mu, \gamma] \subsetneq\left[\mu, \nu_{2}\right] \cap[\mu, \gamma]$. Moreover, if $\mu \sim \gamma$, then

$$
\rho_{[\mu, \gamma]}(\gamma)=\frac{V_{\gamma, \mu}+1}{V_{\mu, \mu}} .
$$

Proof. Note first that since $\rho_{[\gamma, \mu]}(\nu) \rho_{[\mu, \gamma]}(\nu)=1$ for every $\nu$, the claim holds for $\rho_{[\gamma, \mu]}$ exactly when it holds for $\rho_{[\mu, \gamma]}$. If the claim holds whenever $\mu$ and $\gamma$ are adjacent vertices, then we get the desired result by induction on the distance of $\mu$ and $\gamma$, as

$$
\rho_{[\mu, \gamma]}(\nu)=\rho_{[\mu, \eta]}(\nu) \rho_{[\eta, \gamma]}(\nu) .
$$

Hence, it is enough to consider the cases where $\mu \sim \gamma$. We proceed by using induction on the number of vertices of the dual graph.

If $\Gamma$ consists of only two adjacent vertices, then

$$
V=\left[\begin{array}{ll}
1 & 1 \\
1 & 2
\end{array}\right]
$$

and the case is clear.

Suppose that $\Gamma=\Gamma^{\prime}(\eta, U)$ and that the claim holds on the graph $\Gamma^{\prime}$. Note that the valuation matrix of $\Gamma^{\prime}$ is just a restriction of that of $\Gamma$. Moreover, since the valuation matrix of $\Gamma$ is $V^{\mathrm{T}}=V=\left(P^{\mathrm{T}} P\right)^{-1}$, we see that $P_{\eta} V_{\gamma}=q_{\gamma, \eta}$, i. e.,

$$
V_{\gamma, \eta}=\sum_{i \prec \eta} V_{\gamma, i}+\delta_{\eta, \gamma}
$$

If now $\eta \notin[\mu, \gamma]$, then $\rho_{[\mu, \gamma]}(\nu)$ remains unaltered when $\nu \neq \eta$, and if $\nu=\eta$ then $j \in \Gamma_{\gamma}^{\mu}$ for any $j \prec \eta$ exactly when $\eta \in \Gamma_{\gamma}^{\mu}$. Therefore for any $j \prec \eta$,

$$
\rho_{[\mu, \gamma]}(\eta)=\frac{\sum_{i \prec \eta} V_{\gamma, i}}{\sum_{i \prec \eta} V_{\mu, i}}=\rho_{[\mu, \gamma]}(j) .
$$


It remains to show that if $\mu \sim \eta$, then the claim holds for $\rho_{[\mu, \eta]}$, too. If $U=\{\mu\}$ and $\nu \neq \eta$, then by Equation (7) we see that $V_{\eta, \nu}=V_{\mu, \nu}$, and further,

$$
\rho_{[\mu, \eta]}(\nu)=\frac{V_{\eta, \mu}}{V_{\mu, \mu}}<\frac{V_{\eta, \mu}+1}{V_{\mu, \mu}}=\frac{V_{\eta, \eta}}{V_{\mu, \eta}}=\rho_{[\mu, \eta]}(\eta),
$$

as wanted. Especially, Equation (6) holds in this case.

Suppose then that $U=\{\mu, \gamma\}$. Together with (6) Equation (7) yields

$$
\begin{aligned}
\rho_{[\mu, \eta]}(\gamma) & =\frac{V_{\mu, \gamma}+V_{\gamma, \gamma}}{V_{\mu, \gamma}}=1+\frac{V_{\gamma, \mu}+1}{V_{\mu, \mu}} \\
& =1+\frac{V_{\gamma, \gamma}+V_{\gamma, \mu}+1}{V_{\mu, \gamma}+V_{\mu, \mu}}=\frac{V_{\mu, \eta}+V_{\gamma, \eta}+1}{V_{\mu, \eta}}=\rho_{[\mu, \eta]}(\eta) .
\end{aligned}
$$

Moreover,

$$
\rho_{[\mu, \eta]}(\eta)=\rho_{[\mu, \eta]}(\gamma)=\frac{V_{\eta, \gamma}}{V_{\mu, \gamma}}=\frac{V_{\eta, \mu}+V_{\eta, \gamma}+1}{V_{\mu, \mu}+V_{\mu, \gamma}}=\frac{V_{\eta, \mu}+1}{V_{\mu, \mu}}>\rho_{[\mu, \eta]}(\mu) .
$$

This shows that Equation (6) holds for $\eta$. Since

$$
\rho_{[\mu, \eta]}(\nu)=\frac{V_{\mu, \nu}+V_{\gamma, \nu}}{V_{\mu, \nu}}=1+\rho_{[\mu, \gamma]}(\nu)
$$

for every $\nu \neq \eta$, we see that $\rho_{[\mu, \eta]}$ stays constant on any path going away from $[\mu, \eta]$. Hence the claim holds for $\rho_{[\mu, \eta]}$, too.

Proposition 11. Write $\mathbf{1}_{i}=\left(\delta_{i, j}\right)_{j \in \Gamma}$. For any vertices $\gamma, \mu$ and $\eta$, set

$$
\widehat{r}_{[\mu, \gamma]}:=\mathbf{1}_{\gamma}-\rho_{[\mu, \gamma]}(\mu) \mathbf{1}_{\mu} \quad \text { and } \quad \varphi_{\eta}(\nu)=\varphi_{\eta}^{[\mu, \gamma]}(\nu):=\frac{\left(\widehat{r}_{[\mu, \gamma]} V\right)_{\nu}}{V_{\eta, \nu}} .
$$

Then $\varphi_{\eta}(\nu) \geq 0$, where the inequality is strict if and only if $\nu \in \Gamma_{\gamma}^{\mu}$. If $\nu, \nu^{\prime} \in[\mu, \gamma]$ and $d(\mu, \nu)<d\left(\mu, \nu^{\prime}\right)$, then

$$
\varphi_{\eta}(\nu)<\varphi_{\eta}\left(\nu^{\prime}\right) \text {. }
$$

Further, if $\eta \in[\mu, \gamma]$ then $\varphi_{\eta}(\nu)$ is constant on any path intersecting $[\mu, \gamma]$ at most on one point.

Proof. We have

$$
\varphi_{\eta}(\nu)=\rho_{[\eta, \gamma]}(\nu)-\rho_{[\mu, \gamma]}(\mu) \rho_{[\eta, \mu]}(\nu)=\left(\rho_{[\mu, \gamma]}(\nu)-\rho_{[\mu, \gamma]}(\mu)\right) \rho_{[\eta, \mu]}(\nu)
$$

By Lemma $10 \rho_{[\mu, \gamma]}(\nu) \geq \rho_{[\mu, \gamma]}(\mu)$ and thereby also $\varphi_{\eta}(\nu) \geq 0$, where the equality holds exactly when $\nu \in \Gamma \backslash \Gamma_{\gamma}^{\mu}$.

Suppose $\nu, \nu^{\prime} \in[\mu, \gamma]$ and $d(\mu, \nu)<d\left(\mu, \nu^{\prime}\right)$. If $\left.] \mu, \eta\right] \cap[\mu, \gamma]=\emptyset$, then $\rho_{[\eta, \mu]}(\nu)$ does not depend on $\nu \in[\mu, \gamma]$, and again by Lemma 10 we know that $\rho_{[\mu, \gamma]}(\nu)$ is strictly increasing on the path going from $\mu$ to $\gamma$, which proves the case. If $] \mu, \eta] \cap[\mu, \gamma] \neq \emptyset$, then $\rho_{[\eta, \gamma]}(\nu)$ is strictly increasing on $[\eta, \gamma]$ and $\rho_{[\eta, \mu]}(\nu)$ is strictly decreasing on $[\mu, \eta]$, while $\rho_{[\mu, \gamma]}(\mu)$ is a constant. Therefore

$$
\varphi_{\eta}(\nu)=\rho_{[\eta, \gamma]}(\nu)-\rho_{[\mu, \gamma]}(\mu) \rho_{[\eta, \mu]}(\nu)<\rho_{[\eta, \gamma]}\left(\nu^{\prime}\right)-\rho_{[\mu, \gamma]}(\mu) \rho_{[\eta, \mu]}\left(\nu^{\prime}\right)=\varphi_{\eta}\left(\nu^{\prime}\right) .
$$

The rest is now clear. 
In the sequel, we shall make use of the above especially in situations where we have a divisor $F$ with $\xi=\xi_{F}=\lambda(F, D ; \mu)$ for a vertex $\mu$ and we want to modify either $F$ or $D$ or both in such a way that we still have $\xi=\lambda\left(F^{\prime}, D^{\prime} ; \mu\right) \leq$ $\lambda\left(F^{\prime}, D^{\prime} ; \nu\right)$ for every $\nu \in \Gamma$. For that we introduce a modified factorization vector: Let $\widehat{f}, \widehat{g}, \widehat{h} \in \mathbb{Q}^{\Gamma}$ and let $\mu \in \Gamma$. We concentrate on $\mu$ and the vertices adjacent to it and modify $\widehat{f}$ with $\widehat{g}$ and $\widehat{h}$ so that we 'bring' factors $\widehat{g}_{i}$ from each branch emanating from $\mu$ to the closest vertex adjacent to $\mu$ and 'distribute' the factor $\sum_{\nu \sim \mu} \rho_{[\mu, \nu]}(\mu) \widehat{h}_{\nu}$ from $\mu$ to the adjacent vertices.

Notation. Let us write $\widehat{f}_{\langle\widehat{g}\rangle[\widehat{h}]}^{\mu}$ or, if the vertex $\mu$ is clear from the context, just

$$
\widehat{f}_{\langle\widehat{g}\rangle[\widehat{h}]}:=\widehat{f}-\sum_{i \sim \mu} \sum_{j \in \Gamma_{i}^{\mu}} \widehat{g}_{j} \widehat{r}_{[i, j]}+\sum_{i \sim \mu} \widehat{h}_{i} \widehat{r}_{[\mu, i]} .
$$

If either $\widehat{g}$ or $\widehat{h}$ is zero, we may omit it in the notation. Let us also set

$$
\widehat{f}^{\mathcal{N}}:=\widehat{f}-\sum_{i \in \Gamma} \widehat{f}_{i} \widehat{r}_{[\mu, i]} .
$$

Remark 12. Suppose $\widehat{F}=\widehat{f}_{\langle\widehat{g}\rangle[\widehat{h}]}$. Then obviously $\widehat{f}=\widehat{F}_{\langle-\widehat{g}\rangle[-\widehat{h}]}$. Moreover, we have

$$
\widehat{f}^{\mathcal{N}}=\widehat{f}_{\langle\widehat{f}\rangle\left[-\widehat{f}_{\langle\widehat{f}\rangle}\right]} \text {, i. e., } \widehat{f}_{\left[\widehat{f}_{\langle\widehat{f}\rangle}\right.}^{\mathcal{N}}=\widehat{f}_{\langle\widehat{f}\rangle} \text {. }
$$

Lemma 13. Let $f E=\widehat{f} \widehat{E}$ be a divisor. Write $U_{i}:=\Gamma_{i}^{\mu} \backslash\{i\}$. Then

$$
\left(\widehat{f}_{\langle\widehat{g}\rangle[\widehat{h}]}\right)_{i}= \begin{cases}\widehat{f}_{\mu}-\sum_{\nu \sim \mu} \rho_{[\mu, \nu]}(\mu) \widehat{h}_{\nu} & \text { if } i=\mu \\ \widehat{f}_{i}+\widehat{h}_{i}+\sum_{j \in U_{i}} \rho_{[i, j]}(\mu) \widehat{g}_{j} & \text { if } i \sim \mu \\ \widehat{f}_{i}-\widehat{g}_{i} & \text { otherwise. }\end{cases}
$$

Furthermore,

$$
\left(\widehat{f}_{\langle\widehat{g}\rangle} V\right)_{i}=f_{i}-\sum_{\nu \sim \mu} \sum_{j \in \Gamma_{\nu}^{\mu}} \widehat{g}_{j} \varphi_{\mu}^{[\nu, j]}(i) V_{\mu, i}
$$

It follows that if $\widehat{g} \geq 0$, then $\left(\widehat{f}_{\langle\widehat{g}\rangle} V\right)_{i} \leq f_{i}$, where the equality holds when $d(\mu, i) \leq 1$, or more precisely, the inequality is strict exactly when $i \in \Gamma_{j}^{\nu}$ for some $\nu \sim \mu$ and some $j \in \Gamma_{\nu}^{\mu}$ with $\widehat{g}_{j}>0$.

Similarly,

$$
\left(\widehat{f}_{[\widehat{h}]} V\right)_{i}=f_{i}+\sum_{\nu \sim \mu} \widehat{h}_{\nu} \varphi_{\mu}^{[\mu, \nu]}(i) V_{\mu, i}
$$

and if $\widehat{h} \geq 0$, then $\left(\widehat{f}_{[\widehat{h}]} V\right)_{i} \geq f_{i}$, where the inequality is strict exactly when $i \in \Gamma_{\nu}^{\mu}$ for such $\nu \sim \mu$ that $\widehat{h}_{\nu}>0$. 
Proof. Recall that $\widehat{r}_{[i, i]}=0$ for any $i$. A straightforward calculation shows that

$$
\begin{aligned}
\widehat{f}_{\langle\widehat{g}\rangle[\widehat{h}]} & =\widehat{f}-\sum_{i \sim \mu} \sum_{j \in U_{i}} \widehat{g}_{j}\left(\mathbf{1}_{j}-\rho_{[i, j]}(i) \mathbf{1}_{i}\right)+\sum_{i \sim \mu} \widehat{h}_{i}\left(\mathbf{1}_{i}-\rho_{[\mu, i]}(\mu) \mathbf{1}_{\mu}\right) \\
& =\widehat{f}-\sum_{i \sim \mu} \rho_{[\mu, i]}(\mu) \widehat{h}_{i} \mathbf{1}_{\mu}+\sum_{i \sim \mu}\left(\widehat{h}_{i}+\sum_{j \in U_{i}} \rho_{[i, j]}(\mu) \widehat{g}_{j}\right) \mathbf{1}_{i}-\sum_{i \sim \mu} \sum_{j \in U_{i}} \widehat{g}_{j} \mathbf{1}_{j}
\end{aligned}
$$

as $\rho_{[i, j]}(i)=\rho_{[i, j]}(\mu)$ by Lemma 10 . This proves the first assertion. Furthermore, by Proposition 11 we observe that

$$
\left(\widehat{f}_{\langle\widehat{g}\rangle} V\right)_{i}=f_{i}-\sum_{\nu \sim \mu} \sum_{j \in \Gamma_{\nu}^{\mu}} \widehat{g}_{j}\left(\widehat{r}_{[\nu, j]} V\right)_{i}=f_{i}-\sum_{\nu \sim \mu} \sum_{j \in \Gamma_{\nu}^{\mu}} \widehat{g}_{j} \varphi_{\mu}^{[\nu, j]}(i) V_{\mu, i}
$$

where $\varphi_{\mu}^{[\nu, j]}(i) \geq 0$ is positive if and only if $i \in \Gamma_{j}^{\nu}$. Assuming $\widehat{g} \geq 0$, this shows that

$$
\sum_{\nu \sim \mu} \sum_{j \in \Gamma_{\nu}^{\mu}} \widehat{g}_{j} \varphi_{\mu}^{[\nu, j]}(i) V_{\mu, i}>0
$$

if and only if $i \in \Gamma_{j}^{\nu}$ for some $\nu \sim \mu$ and some $j \in \Gamma_{\nu}^{\mu}$ with $\widehat{g}_{j}>0$.

Similarly, by Proposition 11 we get

$$
\left(\widehat{f}_{[\widehat{h}]} V\right)_{i}=f_{i}+\sum_{\nu \sim \mu} \widehat{h}_{\nu}\left(\widehat{r}_{[\mu, \nu]} V\right)_{i}=f_{i}+\sum_{\nu \sim \mu} \widehat{h}_{\nu} \varphi_{\mu}^{[\mu, \nu]}(i) V_{\mu, i},
$$

where $\varphi_{\mu}^{[\mu, \nu]}(i)>0$ exactly when $i \in \Gamma_{\nu}^{\mu}$. Thus, if $\widehat{h} \geq 0$,

$$
\sum_{\nu \sim \mu} \widehat{h}_{\nu} \varphi_{\mu}^{[\mu, \nu]}(i) V_{\mu, i}>0
$$

exactly when $i \in \Gamma_{\nu}^{\mu}$ for such $\nu \sim \mu$ that $\widehat{h}_{\nu}>0$.

Lemma 14. For any divisor $\widehat{f} \widehat{E}$ we have

$$
\widehat{f}^{\mathcal{N}}=\sum_{i \in \Gamma} \rho_{[\mu, i]}(\mu) \widehat{f}_{i} \mathbf{1}_{\mu} \text { and }\left(\widehat{f}^{\mathcal{N}} V\right)_{\mu}=(\widehat{f} V)_{\mu} .
$$

Furthermore, if for some divisor $\widehat{g} \widehat{E}$ holds $\lambda(\widehat{f} \widehat{E}, D ; \mu)=\lambda(\widehat{g} \widehat{E}, D ; \mu)$, then

$$
\widehat{f}^{\mathcal{N}}=\widehat{g}^{\mathcal{N}} \text {. }
$$

Proof. The first equality comes straightforwardly from the definition. A direct calculation shows that

$$
\left(\widehat{f}^{\mathcal{N}} V\right)_{\mu}=\sum_{i \in \Gamma} \rho_{[\mu, i]}(\mu) \widehat{f}_{i} V_{\mu, \mu}=\sum_{i \in \Gamma} \frac{V_{i, \mu}}{V_{\mu, \mu}} \widehat{f}_{i} V_{\mu, \mu}=\sum_{i \in \Gamma} \widehat{f}_{i} V_{i, \mu}=(\widehat{f} V)_{\mu},
$$

as wanted.

Suppose next that $\lambda(\widehat{f} \widehat{E}, D ; \mu)=\lambda(\widehat{g} \widehat{E}, D ; \mu)$. Then $(\widehat{f} V)_{\mu}=(\widehat{g} V)_{\mu}$. By the above we have $\left(\widehat{f}^{\mathcal{N}} V\right)_{\mu}=\left(\widehat{g}^{\mathcal{N}} V\right)_{\mu}$, and further, $\widehat{f}_{\mu}^{\mathcal{N}} V_{\mu, \mu}=\widehat{g}_{\mu}^{\mathcal{N}} V_{\mu, \mu}$. This is to say that $\widehat{f}_{\mu}^{\mathcal{N}}=\widehat{g}_{\mu}^{\mathcal{N}}$, but then $\widehat{f}^{\mathcal{N}}=\widehat{g}^{\mathcal{N}}$. 
Lemma 15. For antinef divisors $f E \neq 0$ and $G$ and for any vertex $\nu \in \Gamma$

$$
\lambda\left(G, \widehat{f}_{\langle\widehat{f}\rangle} \widehat{E} ; \nu\right) \geq \lambda(G, f E ; \nu),
$$

where the equality holds exactly when either $d(\mu, \nu) \leq 1$ or when $\widehat{f}_{j}=0$ for every $j \in \Gamma_{\nu}^{i}$, where $i$ is the vertex in $[\mu, \nu[$ adjacent to $\mu$.

Proof. By Lemma 13 we know that $\widehat{f}_{\langle\widehat{f}\rangle} V_{\nu} \leq f_{\nu}$, where the equality holds exactly when either $d(\mu, \nu) \leq 1$ or when $\widehat{f}_{j}=0$ for every $j \in \Gamma_{\nu}^{i}$, where $i$ is the vertex in $[\mu, \nu[$ adjacent to $\mu$. Thus the claim is clear.

Lemma 16. Let $F=f E$ be an antinef divisor and suppose $0 \neq \widehat{d} \in \mathbb{Q}_{\geq 0}^{\Gamma}$. If

$$
\min _{\nu} \lambda\left(F, \widehat{d}_{\langle\widehat{d}\rangle} \widehat{E} ; \nu\right)=\lambda\left(F, \widehat{d}_{\langle\widehat{d}\rangle} \widehat{E} ; \mu\right),
$$

then we can find an antinef divisor $G$ satisfying

$$
\min _{\nu} \lambda(G, d E ; \nu)=\lambda(G, d E ; \mu)=\lambda(F, d E ; \mu) .
$$

Proof. Observe that for any divisors $U, V \in \Lambda_{\mathbb{Q}}$ and for any nonzero $n \in \mathbb{Q}$,

$$
\lambda(U, V ; \nu)=n \lambda(U, n V ; \nu) .
$$

Thus it is not a restriction to assume that both $\widehat{d}$ and $\widehat{d}_{\langle\widehat{d}\rangle}$ are in $\mathbb{N}^{\Gamma}$.

We need to show that there is a suitable vector $a \in \mathbb{N}^{\Gamma}$, for which the divisor $G=a E$ is as wanted. For $\nu$ with $d(\mu, \nu) \leq 1$ we set $a_{\nu}=f_{\nu}$, so that by Lemma 15 we get $\lambda\left(F, \widehat{d}_{\langle\widehat{d}\rangle} \widehat{E} ; \nu\right)=\lambda\left(a_{\nu}, d E ; \nu\right)$. It follows that $\lambda\left(a_{\nu}, d E ; \nu\right) \geq$ $\lambda\left(a_{\mu}, d E ; \mu\right)$ and

$$
\widehat{a}_{\mu}:=w_{\Gamma}(\mu) a_{\mu}-\sum_{\nu \sim \mu} a_{\nu}=\widehat{f}_{\mu} \geq 0 .
$$

For any branch $\Gamma_{\gamma}^{\mu}$ with $\gamma \sim \mu$ we have two possible cases: either $\widehat{d}_{\eta}=0$ for every $\eta \in \Gamma_{\gamma}^{\mu}$, or $\widehat{d}_{\eta}>0$ for some $\eta \in \Gamma_{\gamma}^{\mu}$. In the first case, we see by Lemma 15 that $\lambda\left(F, \widehat{d}_{\langle\widehat{d}\rangle} \widehat{E} ; \nu\right)=\lambda(F, d E ; \nu)$ for $\nu \in \Gamma_{\gamma}^{\mu}$. Hence we may choose $a_{\nu}=f_{\nu}$ for every $\nu \in \Gamma_{\gamma}^{\mu}$, so that $\lambda\left(a_{\nu}, d E ; \nu\right) \geq \lambda\left(a_{\mu}, d E ; \mu\right)$ and $\widehat{a}_{\nu}:=w_{\Gamma}(\nu) a_{\nu}-$ $\sum_{i \sim \nu} a_{i} \geq 0$. In the latter case, it may happen that $\lambda(F, d E ; \nu)<\lambda(F, d E ; \mu)$ for some $\nu \in \Gamma_{\gamma}^{\mu} \backslash\{\gamma\}$, so that the integers $f_{\nu}$ for $\nu \in \Gamma_{\gamma}^{\mu} \backslash\{\gamma\}$ won't do. Therefore we must apply Lemma 7 in selecting suitable set of integers. If $\lambda(F, d E ; \gamma)>\lambda(F, d E ; \mu)$, then this would be straightforward, since then we could by Lemma 7 choose integers $a_{\nu}$ for $\nu \in \Gamma_{\gamma}^{\mu} \backslash\{\gamma\}$ so that $\lambda\left(a_{\nu}, d E, \nu\right)$ strictly increases on every path in $\Gamma_{\gamma}^{\mu}$ going away from $\gamma$, and $\widehat{a}_{\nu} \geq 0$. Recall that by Lemma 8 we can apply Lemma 7,2$)$ to end vertices, too. In general, we may by using lemma 7 find such integers $a_{\nu}$ for $\nu \in \Gamma_{\gamma}^{\mu} \backslash\{\gamma\}$, that $\lambda\left(a_{\nu}, d E, \nu\right)$ is increasing on the path $[\mu, \eta]$, and strictly increases on every path in $\Gamma_{\gamma}^{\mu}$ going away from $[\mu, \eta]$, and $\widehat{a}_{\nu} \geq 0$. This can be shown as follows.

Let $\eta \in \Gamma_{\gamma}^{\mu}$ be such that $\widehat{d}_{\eta}>0$, and write $\mu=\eta_{0}$ and $\gamma=\eta_{1}$. We have a path of adjacent vertices $\eta_{0} \sim \cdots \sim \eta_{k}=\eta$ for some positive integer $k$. Since $\lambda\left(a_{\eta_{1}}, d E ; \eta_{1}\right) \geq \lambda\left(a_{\eta_{0}}, d E ; \eta_{0}\right)$, we may by Lemma 7 choose integers $a_{\nu}$ 
for $\eta_{0} \neq \nu \sim \eta_{1}$ so that $\widehat{a}_{\eta_{1}} \geq 0$ and $\lambda\left(a_{\nu}, d E ; \nu\right) \geq \lambda\left(a_{\eta_{1}}, d E ; \eta_{1}\right)$, where the equality takes place only if $\nu=\eta_{2}$. Similarly, if $0<i \leq k$ and $\lambda\left(a_{\eta_{i}}, d E ; \eta_{i}\right) \geq$ $\lambda\left(a_{\eta_{i-1}}, d E ; \eta_{i-1}\right)$, we may by Lemma 7 choose integers $a_{\nu}$ for $\eta_{i-1} \neq \nu \sim \eta_{i}$ so that $\widehat{a}_{\eta_{i}} \geq 0$ and $\lambda\left(a_{\nu}, d E ; \nu\right) \geq \lambda\left(a_{\eta_{i}}, d E ; \eta_{i}\right)$, where the equality takes place only if $i<k$ and $\nu=\eta_{i+1}$.

If $\theta \sim \eta_{i}$ for some $i \in\{1, \ldots, k\}$ and $\theta \notin[\mu, \eta]$, then we have

$$
\lambda\left(a_{\theta}, d E ; \theta\right)>\lambda\left(a_{\eta_{i}}, d E ; \eta_{i}\right) \geq \lambda\left(a_{\mu}, d E ; \mu\right) .
$$

Again by using Lemma 7 we may choose integers $a_{\nu}$ for $\nu \in \Gamma_{\theta}^{\eta_{i}}$ so that $\widehat{a}_{\nu} \geq 0$ and $\lambda\left(a_{\nu}, d E ; \nu\right)>\lambda\left(a_{\theta}, d E ; \theta\right)$. Subsequently, by applying Lemma 7 (and Lemma 8), we may find a collection of non-negative integers which meets the requirements of [6, Theorem 1]. Thereby we obtain the desired vector.

Remark 17. By Equation (8) at the beginning of the proof of Lemma 16 we see that $\xi \in \mathcal{H}_{\mu}^{\mathfrak{a}^{n}}$ if and only if $n \xi \in \mathcal{H}_{\mu}^{\mathfrak{a}}$. Thus we may always consider powers $\mathfrak{a}^{n}$ with $n \in \mathbb{N}$ big enough to achieve the situation where both $\widehat{d}$ and $\widehat{d}_{\langle\widehat{d}\rangle}$ are in $\mathbb{N}^{\Gamma}$.

Lemma 18. Let $\mathfrak{a}$ be an ideal with a factorization vector $\widehat{d}$. Suppose $\mathfrak{a}$ is such that $\widehat{d}_{\langle\widehat{d}\rangle} \in \mathbb{N}^{\Gamma}$ and let $\mathfrak{b}$ be the ideal corresponding to it. Then $\xi \in \mathcal{H}_{\mu}^{\mathfrak{a}}$ if and only if $\xi \in \mathcal{H}_{\mu}^{\mathfrak{b}}$.

Proof. If $\xi \in \mathcal{H}_{\mu}^{\mathfrak{a}}$, then there is an antinef divisor $F$ with $\xi_{F}=\lambda(F, D ; \mu)$. It follows from Lemma 13 that $\xi=\lambda(F, D ; \mu)=\lambda\left(F, \widehat{d}_{\langle\widehat{d}\rangle} \widehat{E} ; \mu\right) \leq \lambda\left(F, \widehat{d}_{\langle\widehat{d}\rangle} \widehat{E} ; \nu\right)$, as $\left(\widehat{d}_{\langle\widehat{d}\rangle} V\right)_{i} \leq d_{i}$ where the equality holds for $i$ with $d(\mu, i) \leq 1$. This means that $\xi \in \mathcal{H}_{\mu}^{\mathfrak{b}}$.

If $\xi \in \mathcal{H}_{\mu}^{\mathfrak{b}}$, then $\xi=\lambda\left(F, \widehat{d}_{\langle\widehat{d}\rangle} ; \mu\right) \leq \lambda\left(F, \widehat{d}_{\langle\widehat{d}\rangle} ; \nu\right)$, then by Lemma 16 we have such an antinef divisor $G$ that $\xi=\lambda(G, D ; \mu) \leq \lambda(G, D ; \nu)$, which shows that $\xi \in \mathcal{H}_{\mu}^{\mathfrak{a}}$.

\section{Semigroup of values}

Let $\mathcal{S} \mathcal{V}_{\mu}=\left(\mathcal{S} \mathcal{V}_{\mu},+\right)$ be the submonoid of $\mathbb{N}$ generated by values $V_{\mu, i}, i \in \Gamma$. This is called the value semigroup of $v_{\mu}$. Recall that if $\Gamma$ is the dual graph of the simple ideal $\mathfrak{p}_{\mu}$, then the Zariski exponents are the values of the form $V_{\mu, \tau}$ where $\tau$ is an end (see, e. g., [8, Remark 6.6]). In general, with any dual graph, we may consider values $V_{\mu, \tau}$ where $\tau$ is an end of the graph. We then get the following:

Proposition 19. Let $\Gamma$ be a dual graph containing $\mu$. As a submonoid of $\mathbb{N}$, the semigroup $\mathcal{S V}_{\mu}$ is always generated by the set of Zariski exponents of $\mu$, i. e., the values

$$
\left\{V_{\mu, \tau_{i}} \mid i=0 \text {, or } i=1, \ldots, g+1 \text { and } \tau_{i} \neq \mu\right\},
$$

where $\tau_{0}$ is the root and the indices $\tau_{1}, \ldots, \tau_{g+1}$ are as in Definition 2. In general, we may write

$$
\mathcal{S} \mathcal{V}_{\mu}=\left\langle V_{\mu, \tau} \mid v_{\Gamma}(\tau) \leq 1\right\rangle
$$


Proof. If $\mu$ is the only vertex, then $\mathcal{S} \mathcal{V}_{\mu}=\left\langle V_{\mu, \mu}\right\rangle$, and the claim is clear. Suppose that $\Gamma=\Gamma^{\prime}(\eta, U)$. If there is $\eta^{\prime} \in \Gamma$ different from $\eta$, for which $w_{\Gamma}\left(\eta^{\prime}\right)=1$, then we may find a graph $\Gamma^{\prime \prime}$ containing $\eta$, for which $\Gamma=\Gamma^{\prime \prime}\left(\eta^{\prime}, U^{\prime}\right)$. Thus we may in this situation choose $\eta \neq \mu$. But if $\eta \neq \mu$, then

$$
V_{\mu, \eta}=\sum_{\nu \sim \eta} V_{\mu, \nu}
$$

and so the value semigroup remains unchanged under the blowup. Hence we may assume that $\eta=\mu$ is the only vertex with weight one. This means that $\Gamma$ is the dual graph of the minimal principalization of the simple ideal corresponding to the vertex $\mu$, and the claim follows from Lemma 20 below.

Value semigroups are closely related to the jumping numbers. It follows from [8, Theorem 6.2] (see also [8, Lemma 6.1 and Remark 6.6]) that if $\mathfrak{a}=\mathfrak{p}_{\mu}$ is simple, then $\xi$ is a jumping number of $\mathfrak{a}$ supported at $\mu$ if and only if

$$
\xi V_{\mu, \mu}-q_{\mu, \gamma}-V_{\mu, \tau} \in\left\langle q_{\mu, \gamma}, V_{\mu, \tau}\right\rangle,
$$

where $(\gamma, \tau)$ is the pair associated to $\mu$ (Definition 2). Our aim is to generalize this formula. Observe here that the first one of the two generators is not necessarily in $\mathcal{S} \mathcal{V}_{\mu}$, but as we shall see in Proposition 21 below, $q_{\mu, \gamma}$ is the greatest common divisor of the values $V_{\mu, i}$ with $i \in \Gamma_{\gamma}^{\mu}$, while $V_{\mu, \tau}$ is that of the values coming from the branch $\Gamma_{\tau}^{\mu}$.

Let $\mathfrak{a}$ be a complete ideal in $R$ with a dual graph $\Gamma$. For vertices $\mu, \nu \in \Gamma$, let us define a submonoid $\left(\mathcal{S} \mathcal{V}_{\nu}^{\mu},+\right)$ of $\mathcal{S} \mathcal{V}_{\mu}$ corresponding to the branch $\Gamma_{\nu}^{\mu}$ by setting

$$
\mathcal{S} \mathcal{V}_{\nu}^{\mu}:=\left\langle V_{\mu, i} \mid i \in \Gamma_{\nu}^{\mu} \cup\{\mu\}\right\rangle .
$$

Clearly, $\mathcal{S} \mathcal{V}_{\nu}^{\mu}=\mathcal{S} \mathcal{V}_{\eta}^{\mu}$, if $\nu$ and $\eta$ define the same branch. Set $s_{\nu}^{\mu}:=\operatorname{gcd} \mathcal{S} \mathcal{V}_{\nu}^{\mu}$ and write $\left(S^{\mu},+\right)$ for the submonoid generated by these numbers, so that

$$
S^{\mu}:=\left\langle s_{\nu}^{\mu} \mid \nu \in \Gamma\right\rangle
$$

Note that if $\mu$ is the only vertex in $\Gamma$, then $S^{\mu}=\left\langle s_{\mu}^{\mu}\right\rangle=\mathbb{N}$. Otherwise $S^{\mu}=\left\langle s_{\nu}^{\mu} \mid \nu \sim \mu\right\rangle$.

Lemma 20. Let $\Gamma$ be the dual graph of the minimal principalization of a simple ideal $\mathfrak{p}_{\mu}$ corresponding to the vertex $\mu$. Let $\left(\gamma_{0}, \tau_{1}\right), \ldots,\left(\gamma_{g}, \tau_{g+1}\right)=(\gamma, \tau)$ be the sequence of pairs associated to $\mu$ and write $\tau_{0}=\gamma_{0}$ for the root. Then

$$
\mathcal{S} \mathcal{V}_{\gamma}^{\mu}=\left\langle V_{\mu, \tau_{0}}, \ldots, V_{\mu, \tau_{g}}\right\rangle \text { and } \mathcal{S} \mathcal{V}_{\tau}^{\mu}=\left\langle V_{\mu, \tau}\right\rangle \text {. }
$$

Furthermore, we have $s_{\gamma}^{\mu}=q_{\mu, \gamma}$ and $s_{\tau}^{\mu}=V_{\mu, \tau}$, and $s_{\gamma}^{\mu} s_{\tau}^{\mu}=V_{\mu, \mu}$. The greatest integral multiple of $s_{\gamma}^{\mu}$ not in $\mathcal{S} \mathcal{V}_{\gamma}^{\mu}$ is

$$
\sum_{i=1}^{g} V_{\mu, \gamma_{i}}-\sum_{i=0}^{g} V_{\mu, \tau_{i}} .
$$

Proof. By Equation (3) we have for $\nu \in \Gamma \backslash\{\mu\}$

$$
w_{\Gamma}(\nu) V_{\mu, \nu}=\sum_{i \sim \nu} V_{\mu, i}
$$


Subsequently, we observe that if $\eta$ is such that $d(\nu, \eta) \leq 1$ and a nonnegative integer $k$ divides $V_{\mu, i}$ for every $i \neq \eta$ with $d(\nu, i) \leq 1$, then $k$ divides $V_{\mu, \eta}$ also. It follows that

$$
\left\langle V_{\mu, i} \mid i \in \Gamma_{\tau_{n}}^{\gamma_{n}} \cup\left\{\gamma_{n}\right\}\right\rangle=\left\langle V_{\mu, \tau_{n}}\right\rangle
$$

as semigroups for any $n=0, \ldots, g+1,\left(\gamma_{g+1}:=\mu\right)$, so that $V_{\mu, \tau_{n}}$ is the greatest common divisor of the set. Especially, $\mathcal{S} \mathcal{V}_{\tau}^{\mu}=\left\langle V_{\mu, \tau}\right\rangle$ so that $s_{\tau}^{\mu}=V_{\mu, \tau}$. Let us define a submonoid $\mathcal{W}_{n}$ as

$$
\mathcal{W}_{n}:=\left\langle V_{\mu, i} \mid i \in \Gamma_{\tau_{0}}^{\gamma_{n}} \cup\left\{\gamma_{n}\right\}\right\rangle
$$

for $n=0, \ldots, g+1$, so that $\mathcal{S} \mathcal{V}_{\gamma}^{\mu}=\mathcal{W}_{g+1}$. Let $\Sigma_{n}$ stand for the greatest integral multiple of gcd $\mathcal{W}_{n}$ not in $\mathcal{W}_{n}$. Clearly, $\mathcal{W}_{0}=\left\langle V_{\mu, \tau_{0}}\right\rangle$ and $\operatorname{gcd} \mathcal{W}_{0}=V_{\mu, \tau_{0}}$ and $\Sigma_{0}=-V_{\mu, \tau_{0}}$. To complete our proof it is enough to show that for $n=0, \ldots, g$, $(10)$

$\mathcal{W}_{n+1}=\left\langle\mathcal{W}_{n}, V_{\mu, \tau_{n}}\right\rangle, \operatorname{gcd} \mathcal{W}_{n+1}=q_{\mu, \gamma_{n}}$ and $\Sigma_{n+1}=\Sigma_{n}+V_{\mu, \gamma_{n}}-V_{\mu, \tau_{n}}<V_{\mu, \gamma_{n}}$.

At first, note that the vertices of the set $\left(\Gamma_{\tau_{0}}^{\gamma_{n+1}} \cup\left\{\gamma_{n+1}\right\}\right) \backslash\left(\Gamma_{\tau_{0}}^{\gamma_{n}} \cup \Gamma_{\tau_{n}}^{\gamma_{n}}\right)$ yield a path $\gamma_{n}=\eta_{0}^{n} \sim \cdots \sim \eta_{k_{n}}^{n}=\gamma_{n+1}$ for every $n=0, \ldots, g$. Thus if $n=0$, it follows from the observation we made at the beginning of our proof that $V_{\mu, \tau_{0}}$ divides $V_{\mu, \eta_{1}^{0}}$ and subsequently $V_{\mu, \eta_{i}^{0}}$ for every $i=0, \ldots, k_{0}$. Hence $\mathcal{W}_{1}=\left\langle V_{\mu, \tau_{0}}\right\rangle=\left\langle\mathcal{W}_{0}, V_{\mu, \tau_{0}}\right\rangle$ and the greatest common divisor of the set is $q_{\mu, \gamma_{0}}=V_{\mu, \tau_{0}}$. Moreover, $\Sigma_{1}=\Sigma_{0}+V_{\mu, \gamma_{0}}-V_{\mu, \tau_{0}}=-V_{\mu, \gamma_{0}}$ so that $\Sigma_{1}<V_{\mu, \gamma_{0}}$.

Assume that Equation (10) holds if $n<n_{0}$ for some $n_{0} \in\{1, \ldots, g\}$. Suppose then that $n=n_{0}$. Recall that by [8, Lemma 6.1] (see also [8, Remark 6.6]) we have

$$
\operatorname{gcd}\left\{q_{\mu, \gamma_{n-1}}, V_{\mu, \tau_{n}}\right\}=q_{\mu, \gamma_{n}} .
$$

Again, by the observation at the beginning of the proof, we see that $q_{\mu, \gamma_{n}}$ divides $V_{\mu, \nu}$ for every $\nu \neq \eta_{1}^{n}$ with $d\left(\gamma_{n}, \nu\right) \leq 1$, and therefore it divides also $V_{\mu, \eta_{1}^{n}}$. Subsequently, it divides every $V_{\mu, \eta_{i}^{n}}$ with $i=0, \ldots, k_{n}$. This shows that $\operatorname{gcd} \mathcal{W}_{n+1}=q_{\mu, \gamma_{n}}$.

Let us next verify that $\Sigma_{n+1}=\Sigma_{n}+V_{\mu, \gamma_{n}}-V_{\mu, \tau_{n}}$ is the greatest integral multiple of $q_{\mu, \gamma_{n}}$ not in $\left\langle\mathcal{W}_{n}, V_{\mu, \tau_{n}}\right\rangle$. By [8, Corollary 3.16] we know that $q_{\mu, \gamma_{n-1}}=q_{\gamma_{n}, \gamma_{n-1}} q_{\mu, \gamma_{n}}$. Therefore by [8, Lemma 6.1] we get $V_{\mu, \gamma_{n}}=q_{\gamma_{n}, \gamma_{n-1}} V_{\mu, \tau_{n}}$, and so

$$
\Sigma_{n+1}=\Sigma_{n}+\left(q_{\gamma_{n}, \gamma_{n-1}}-1\right) V_{\mu, \tau_{n}}
$$

which is clearly an integral multiple of $q_{\mu, \gamma_{n}}$.

Let $m \in \mathbb{N}$ be such that $\Sigma_{n+1}+m q_{\mu, \gamma_{n}}$ is in $\left\langle\mathcal{W}_{n}, V_{\mu, \tau_{n}}\right\rangle$. Equivalently, we may write $\Sigma_{n+1}+m q_{\mu, \gamma_{n}}=s+t V_{\mu, \tau_{n}}$ for some $s \in \mathcal{W}_{n}$ and $t \in\left\{0, \ldots, q_{\gamma_{n}, \gamma_{n-1}}-1\right\}$. Now $u:=q_{\gamma_{n}, \gamma_{n-1}}-1-t$ belongs to the same set as $t$ and we may reformulate

$$
s=\Sigma_{n}+u V_{\mu, \tau_{n}}+m q_{\mu, \gamma_{n}} \in \mathcal{W}_{n} .
$$

This holds if and only if $u V_{\mu, \tau_{n}}+m q_{\mu, \gamma_{n}}$ is a positive multiple of $q_{\mu, \gamma_{n-1}}$. Observe that the map

$$
\varphi: u \mapsto \frac{u V_{\mu, \tau_{n}}}{q_{\mu, \gamma_{n}}} \bmod q_{\gamma_{n}, \gamma_{n-1}}
$$


is a bijection between the sets $\left\{0, \ldots, q_{\gamma_{n}, \gamma_{n-1}}-1\right\}$ and $\mathbb{Z}_{q_{\gamma_{n}, \gamma_{n-1}}}$ following from the fact that $\operatorname{gcd}\left\{q_{\gamma_{n}, \gamma_{n-1}}, V_{\mu, \tau_{n}} / q_{\mu, \gamma_{n}}\right\}=1$. Therefore we may always find an integer $u \in\left\{0, \ldots, q_{\gamma_{n}, \gamma_{n-1}}-1\right\}$ so that $\varphi(u)=-m \bmod q_{\gamma_{n}, \gamma_{n-1}}$, but then $u V_{\mu, \tau_{n}}+m q_{\mu, \gamma_{n}}$ is divisible by $q_{\mu, \gamma_{n-1}}$, and it is positive if and only if $m>0$. Thus $\Sigma_{n+1}$ is the greatest integral multiple of $q_{\mu, \gamma_{n}}$ which does not belong to $\left\langle\mathcal{W}_{n}, V_{\mu, \tau_{n}}\right\rangle$.

Finally, let us verify that $\Sigma_{n+1}<V_{\mu, \gamma_{n}}$ and that $\mathcal{W}_{n+1}=\left\langle\mathcal{W}_{n}, V_{\mu, \tau_{n}}\right\rangle$. By (1) we get $P_{\nu} V_{\mu}=q_{\mu, \nu}$, and since $P Q=1$ we see that $q_{\mu, \nu}=\sum_{i \succ \nu} q_{\mu, i}+\delta_{\mu, \nu}$ and subsequently $q_{\mu, \nu}>0$ if $\nu \subset \mu$. Hence $V_{\mu, \nu^{\prime}}<V_{\mu, \nu}$ if $\nu^{\prime} \prec \nu \subset \mu$. It follows that $V_{\mu, \nu^{\prime}}<V_{\mu, \nu}$ if $\nu^{\prime} \subset \nu \subset \mu$. Especially, $V_{\mu, \gamma_{n-1}}<V_{\mu, \tau_{n}}$, but then

$$
\Sigma_{n+1}<V_{\mu, \gamma_{n-1}}+V_{\mu, \gamma_{n}}-V_{\mu, \tau_{n}}<V_{\mu, \gamma_{n}} \leq V_{\mu, \eta_{i}^{n}}
$$

for every $i=0, \ldots, k_{n}$. As $\Sigma_{n+1}$ is the greatest integral multiple of $q_{\mu, \gamma_{n}}$ not in $\left\langle\mathcal{W}_{n}, V_{\mu, \tau_{n}}\right\rangle$, we see that $V_{\mu, \eta_{i}^{n}} \in\left\langle\mathcal{W}_{n}, V_{\mu, \tau_{n}}\right\rangle$ for every $i=0, \ldots, k_{n}$, but then

$$
\left\langle\mathcal{W}_{n}, V_{\mu, \tau_{n}}\right\rangle \subset \mathcal{W}_{n+1} \subset\left\langle\mathcal{W}_{n}, V_{\mu, \tau_{n}}\right\rangle
$$

as wanted. Thus Equation (10) holds for any $n=0, \ldots, g$, and the claim follows.

Proposition 21. The submonoid $\mathcal{S} \mathcal{V}_{\nu}^{\mu}$ is generated by those values $V_{\mu, i}$ where $i \in \Gamma_{\nu}^{\mu}$ is an end. More precisely, if $(\gamma, \tau)$ is the pair associated to $\mu$, then the following holds:

$$
\mathcal{S V}_{\nu}^{\mu}= \begin{cases}\left\langle V_{\mu, \mu}\right\rangle & \text { if } \Gamma_{\nu}^{\mu} \text { is posterior to } \mu \\ \left\langle V_{\mu, \tau}\right\rangle & \text { if } \nu \in \Gamma_{\tau}^{\mu} \\ \left\langle V_{\mu, i} \mid v_{\Gamma}(i)=1, i \in \Gamma_{\gamma}^{\mu}\right\rangle & \text { if } \nu \in \Gamma_{\gamma}^{\mu} .\end{cases}
$$

We have $s_{\nu}^{\mu}=q_{\mu, \gamma}$ if $\nu \in \Gamma_{\gamma}^{\mu}$, otherwise $s_{\nu}^{\mu}$ is the generator of the submonoid $\mathcal{S} \mathcal{V}_{\nu}^{\mu}$. For any $\nu \neq \mu$, the greatest integral multiple of $s_{\nu}^{\mu}$ not in $\mathcal{S V}_{\nu}^{\mu}$ is

$$
M_{\nu}=M_{\nu}^{\mu}:=\sum_{j \in \Gamma_{\nu}^{\mu}}\left(v_{\Gamma}(j)-2\right) V_{\mu, j}
$$

whereas $M_{\mu}:=-V_{\mu, \mu}$.

Proof. Suppose that $\eta \neq \mu$ is a vertex in $\Gamma$ with $w_{\Gamma}(\eta)=1$. Let $\Gamma^{\prime}$ be such that $\Gamma=\Gamma^{\prime}(\eta, U)$ and suppose that the claim holds for $\Gamma^{\prime}$. In the case $\nu \in \Gamma^{\prime}$, let $\mathcal{S} \mathcal{V}^{\prime \mu}{ }_{\nu}$ denote the submonoid generated by the values $V_{\mu, i}$ where $i \in\left(\Gamma^{\prime}\right)_{\nu}^{\mu} \cup\{\mu\}$. Write $M_{\nu}^{\prime}$ for the greatest integral multiple of $\operatorname{gcd} \mathcal{S} \mathcal{V}_{\nu}^{\prime \mu}$ not in $\mathcal{S} \mathcal{V}^{\prime \mu}{ }^{\mu}$.

As $U$ consists of vertices adjacent to $\eta$ we observe that if $\eta \in \Gamma_{\nu}^{\mu}$, then $U \subset \Gamma_{\nu}^{\mu} \cup\{\mu\}$. By Equation 3 we then see that if $V_{\mu, i} \in \mathcal{S V}^{\prime \mu}{ }_{\nu}$ for every $i \in U$, then $V_{\mu, \eta} \in \mathcal{S} \mathcal{V}_{\nu}^{\prime \mu}$, as $w_{\Gamma}(\eta)=1$ and $\delta_{\mu, \eta}=0$. Thus the submonoid $\mathcal{S} \mathcal{V}_{\nu}^{\mu}$ is the same as the submonoid $\mathcal{S} \mathcal{V}^{\prime \mu}{ }_{\nu}$ when $\nu \neq \eta$. Further, $\mathcal{S} \mathcal{V}_{\eta}^{\mu}=\mathcal{S} \mathcal{V}_{\nu}^{\mu}$ for any $\nu \in \Gamma_{\eta}^{\mu}$, and in the case $\Gamma_{\eta}^{\mu}=\{\eta\}$ we see that $\mathcal{S} \mathcal{V}_{\eta}^{\mu}=\mathcal{S} \mathcal{V}_{\mu}^{\prime \mu}$, as $\eta$ must be a free blowup of $\mu$, in which case Equation (3) yields $V_{\mu, \eta}=V_{\mu, \mu}$. Subsequently, the greatest common divisor of the values from a branch remains unchanged under the blowup. Obviously, this is also the case with the greatest of its integral multiples not in the submonoid. 
To see that the formula for $M_{\nu}$ holds, note first that, $M_{\nu}=M_{\nu}^{\prime}$ if $\eta \notin \Gamma_{\nu}^{\mu}$. If $\Gamma_{\nu}^{\mu}=\{\eta\}$, then $v_{\Gamma}(\eta)=1$ and $M_{\eta}=\left(v_{\Gamma}(\eta)-2\right) V_{\mu, \eta}=-V_{\mu, \eta}$, as wanted, since $\mathcal{S} \mathcal{V}_{\eta}^{\mu}=\left\langle V_{\mu, \eta}\right\rangle$ and $V_{\mu, \eta}=V_{\mu, \mu}$. Suppose then that $\eta$ is not the only vertex on $\Gamma_{\nu}^{\mu}$. As $M_{\nu}=M_{\nu^{\prime}}$ for every $\nu^{\prime} \in \Gamma_{\nu}^{\mu}$, we may assume that $\nu \neq \eta$. Then

$$
M_{\nu}=M_{\nu}^{\prime}+\Delta_{\eta},
$$

where

$$
\Delta_{\eta}:=\left(v_{\Gamma}(\eta)-2\right) V_{\mu, \eta}-\sum_{\mu \neq i \sim \eta}\left(v_{\Gamma^{\prime}}(i)-v_{\Gamma}(i)\right) V_{\mu, i} .
$$

If $\eta$ is free, then $v_{\Gamma}(\eta)=1$, but since there is only one vertex $i$ adjacent to it and $\eta$ is not the only vertex on the branch, we see that $i \neq \mu, v_{\Gamma}(i)=v_{\Gamma^{\prime}}(i)+1$ and $V_{\mu, \eta}=V_{\mu, i}$ by Equation (3). Therefore $\Delta_{\eta}=0$. If $\eta$ is not free, then $v_{\Gamma}(\eta)=2$ and $v_{\Gamma}(i)=v_{\Gamma^{\prime}}(i)$ for any $i \sim \eta$. Hence $\Delta_{\eta}=0$ in any case. This shows that also the numbers $M_{\nu}$ remain unchanged under the blowup.

By the above we may blow down $\eta$, and continuing this way, we may eventually assume that $w_{\Gamma}(i)>1$ for every vertex $i \neq \mu$. This is to say that $\Gamma$ is the dual graph of the minimal principalization of a simple ideal $\mathfrak{p}_{\mu}$ corresponding to the vertex $\mu$, but this case is clear by Lemma 20 above.

Remark 22. Because $V_{\mu, \mu}=q_{\mu, \gamma} V_{\mu, \tau}$, we see that $S^{\mu}=\left\langle q_{\mu, \gamma}, V_{\mu, \tau}\right\rangle$. Furthermore, $\operatorname{gcd}\left\{q_{\mu, \gamma}, V_{\mu, \tau}\right\}=q_{\mu, \mu}$ by (11). On the other hand, $q_{\mu, \mu}=1$ as easily seen since $Q=P^{-1}$. It follows that $S^{\mu}$ is always a numerical semigroup, i. e., a submonoid of $\mathbb{N}$ with finite complement.

\section{MAIN RESUlT}

Theorem 23. A positive number $\xi$ is a jumping number in $\mathcal{H}_{\mu}^{\mathfrak{a}}$ exactly when

$$
h_{\mu}^{\mathfrak{a}}(\xi):=d_{\mu} \xi+\left(v_{\Gamma}(\mu)-2\right) V_{\mu, \mu}-\sum_{\nu \sim \mu} s_{\nu}^{\mu}\left\lceil\sum_{i \in \Gamma_{\nu}^{\mu}} \frac{\widehat{d_{i}} V_{\mu, i}}{s_{\nu}^{\mu}} \xi\right]^{+} \in S^{\mu},
$$

where $S^{\mu}$ is the submonoid of $\mathbb{N}$ defined by Equation (9) and \lceil\rceil$^{+}$means rounding up to the nearest positive integer.

Remark 24. This result yields a formula for the set of the jumping numbers of $\mathfrak{a}$ supported at $\mu$ :

$$
\mathcal{H}_{\mu}^{\mathfrak{a}}=\left\{\frac{t}{d_{\mu}} \mid t+\left(v_{\Gamma}(\mu)-2\right) V_{\mu, \mu}-\sum_{\nu \sim \mu} s_{\nu}^{\mu}\left\lceil t \sum_{i \in \Gamma_{\nu}^{\mu}} \frac{\widehat{d}_{i} V_{\mu, i}}{s_{\nu}^{\mu} d_{\mu}}\right]^{+} \in S^{\mu}\right\} .
$$

Remark 25. It easily follows from Proposition 21 that the numbers $s_{\nu}^{\mu}$ and $V_{\mu, \mu}$ present in the above formula generate $S^{\mu}$. Moreover,

$$
s_{\nu}^{\mu}=\operatorname{gcd}\left\{V_{\mu, \tau} \mid v_{\Gamma}(\tau)=1 \text { and } \tau \in \Gamma_{\nu}^{\mu}\right\}
$$

for every $\nu \sim \mu$. 
Remark 26. By Proposition $21 V_{\mu, \mu} \in S^{\mu}$ and $s_{\nu}^{\mu}=V_{\mu, \mu}$ for any $\nu$ such that $\Gamma_{\nu}^{\mu}$ is posterior to $\mu$. It follows that, for any positive integers $t_{\nu}$,

$$
\sum_{\nu \sim \mu} s_{\nu}^{\mu} t_{\nu}-\left(v_{\Gamma}(\mu)-2\right) V_{\mu, \mu} \in S^{\mu}
$$

Subsequently, if $\xi \in \mathcal{H}_{\mu}^{\mathfrak{a}}$, then $d_{\mu} \xi \in S^{\mu}$. Note that the converse is not true: if for example the ideal in question is the maximal ideal and $\xi=1$, then $\mu=\tau_{0}$ is the only vertex of the dual graph of our ideal and $d_{\mu}=1$. Clearly, $S^{\mu}=\mathbb{N}$ so that $d_{\mu} \xi \in S^{\mu}$, but as well known, 1 is not a jumping number of the maximal ideal, see e. g. Example 27 below.

Proof of Theorem 23. Note first that the case $\mu$ is the only vertex of the dual graph is trivial and is dealt with in Example 27 below. Thus we may assume $\mu$ has adjacent vertices. Obviously, by Remark 5 we could also choose any dual graph containing $\mu$ and having other vertices, too. To begin with, observe that

$$
h_{\mu}^{\mathfrak{a}}(\xi)=\frac{n d_{\mu} \xi}{n}+\left(v_{\Gamma}(\mu)-2\right) V_{\mu, \mu}-\sum_{\nu \sim \mu} s_{\nu}^{\mu}\left[\sum_{i \in \Gamma_{\nu}^{\mu}} \frac{n \widehat{d}_{i} V_{\mu, i}}{s_{\nu}^{\mu}} \cdot \frac{\xi}{n}\right]^{+}=h_{\mu}^{\mathfrak{a}^{n}}\left(\frac{\xi}{n}\right)
$$

Hence $h_{\mu}^{\mathfrak{a}}(\xi) \in S^{\mu}$ exactly, when $h_{\mu}^{\mathfrak{a}^{n}}\left(\frac{\xi}{n}\right) \in S^{\mu}$. Together with Remark 17 this shows that, by considering $\mathfrak{a}^{n}$ with $n$ big enough, we may assume $\widehat{d}_{\langle\widehat{d}\rangle} \in \mathbb{N}^{\Gamma}$.

Let $\mathfrak{b}$ be the ideal having the factorization vector $\widehat{d}_{\langle\widehat{d}\rangle}$. Note that $\mathfrak{b}$ is a product of ideals $\mathfrak{p}_{\nu}$ where $d(\nu, \mu) \leq 1$, and we may regard $\Gamma$ as a dual graph and $V$ as a valuation matrix of $\mathfrak{b}$ (see Remark 5). According to Lemma 13

$$
\left(\widehat{d}_{\langle\widehat{d}\rangle} V\right)_{\nu}=d_{\nu} \text { and } \sum_{i \in \Gamma_{\nu}^{\mu}}\left(\widehat{d}_{\langle\widehat{d}\rangle}\right)_{i} V_{\mu, i}=\sum_{i \in \Gamma_{\nu}^{\mu}} \widehat{d}_{i} V_{\mu, i}
$$

for $\nu$ with $d(\mu, \nu) \leq 1$. This shows, together with Lemma 18, that we may assume $\mathfrak{a}=\mathfrak{b}$, i. e., $\widehat{d}_{\nu}=0$ unless $d(\mu, \nu) \leq 1$. Thereby

$$
\sum_{i \in \Gamma_{\nu}^{\mu}} \frac{\widehat{d}_{i} V_{\mu, i}}{s_{\nu}^{\mu}} \xi=\frac{\widehat{d}_{\nu} V_{\mu, \nu}}{s_{\nu}^{\mu}} \xi
$$

for every $\nu \sim \mu$.

Set $\widehat{g}:=\xi \widehat{d}+\widehat{\kappa}$, where $\widehat{\kappa}:=\left(v_{\Gamma}(\nu)-2\right)_{\nu \in \Gamma}$. Observe that because

$$
(1, \ldots, 1) P^{\mathrm{T}} P=\left(w_{\Gamma}(\nu)-v_{\Gamma}(\nu)\right)_{\nu \in \Gamma}
$$

and since $\widehat{k}=\left(2-w_{\Gamma}(\nu)\right)_{\nu \in \Gamma}$ by (4), we get $k_{\nu}+1=-(\widehat{\kappa} V)_{\nu}$. Subsequently, we obtain

$$
\lambda(\widehat{g} \widehat{E}, d E ; \nu)=\frac{\xi d_{\nu}+(\widehat{\kappa} V)_{\nu}+k_{\nu}+1}{d_{\nu}}=\xi
$$

for any $\nu \in \Gamma$. Consider a vector $\widehat{\phi}:=\widehat{g}_{\langle\widehat{\kappa}\rangle[\zeta]}$ where $\zeta_{\nu}:=0$ unless $\nu \sim \mu$ in which case

$$
\zeta_{\nu}:=\frac{s_{\nu}^{\mu}}{V_{\mu, \nu}}\left(\left\lceil\frac{\widehat{d}_{\nu} V_{\mu, \nu}}{s_{\nu}^{\mu}} \xi\right\rceil^{+}-\frac{\widehat{d}_{\nu} V_{\mu, \nu}}{s_{\nu}^{\mu}} \xi\right) .
$$


Let $M_{\nu}$ be as in Proposition 21. By using Lemma 13 a direct calculation shows that

$$
\widehat{\phi}_{\mu}=\xi \widehat{d}_{\mu}+\left(v_{\Gamma}(\mu)-2\right)-\sum_{\nu \sim \mu} \rho_{[\mu, \nu]}(\mu) \zeta_{\nu}=\frac{h_{\mu}^{\mathfrak{a}}(\xi)}{V_{\mu, \mu}}
$$

and

$$
\widehat{\phi}_{\nu}=\xi \widehat{d}_{\nu}+\zeta_{\nu}+\sum_{i \in \Gamma_{\nu}^{\mu}} \rho_{[\nu, i]}(\mu)\left(v_{\Gamma}(i)-2\right)=\frac{M_{\nu}}{V_{\mu, \nu}}+\frac{s_{\nu}^{\mu}}{V_{\mu, \nu}}\left\lceil\frac{\widehat{d}_{\nu} V_{\mu, \nu}}{s_{\nu}^{\mu}} \xi\right]^{+} .
$$

Observe that since $\widehat{\phi}=\left(\xi \widehat{d}+\widehat{\kappa}_{\langle\widehat{\kappa}\rangle}\right)_{[\zeta]}$, we have $\widehat{\phi}_{\nu}=0$ when $d(\mu, \nu)>1$. Furthermore, as $\zeta_{\nu} \geq 0$ where the equality may take place only if $\widehat{d}_{\nu}>0$, we know by Lemma 13 that $(\widehat{\phi} V)_{\mu}=(\widehat{g} V)_{\mu}$ and

$$
(\widehat{\phi} V)_{\nu} \geq(\widehat{g} V)_{\nu}
$$

for every $\nu$ with $\nu \sim \mu$, where the equality may take place only if $\widehat{d}_{\nu}>0$.

Suppose now that $\xi \in \mathcal{H}_{\mu}^{\mathfrak{a}}$. By [6, Theorem 1 and Lemma 6] we have $\widehat{f} \in \mathbb{N}^{\Gamma}$ satisfying $\widehat{f}_{\nu}>0$ only if $\nu$ is an end different from $\mu$, and further, $\lambda\left((\widehat{f} V)_{\mu}, \mu\right)=\xi$ and $\lambda\left((\widehat{f} V)_{\nu}, \nu\right)$ is increasing on every path going away from $\mu$. Because also $\lambda\left((\widehat{\phi} V)_{\mu}, \mu\right)=\lambda\left((\widehat{g} V)_{\mu}, \mu\right)=\xi$, we get by applying Lemma $14 \widehat{\phi}^{\mathcal{N}}=\widehat{f}^{\mathcal{N}}$, and further

$$
\widehat{\phi}_{\mu}^{\mathcal{N}} V_{\mu, \mu}=\left(\widehat{\phi}^{\mathcal{N}} V\right)_{\mu}=\left(\widehat{f}^{\mathcal{N}} V\right)_{\mu}=\sum_{\nu \sim \mu} \sum_{i \in \Gamma_{\nu}^{\mu}} \widehat{f}_{i} V_{\mu, i}
$$

Thus Equation 12 gives

$$
h_{\mu}^{\mathfrak{a}}(\xi)=\left(\widehat{\phi}_{\mu}^{\mathcal{N}}-\sum_{\nu \sim \mu} \rho_{[\mu, \nu]}(\mu) \widehat{\phi}_{\nu}\right) V_{\mu, \mu}=\sum_{\nu \sim \mu}\left(\sum_{i \in \Gamma_{\nu}^{\mu}} \widehat{f}_{i} V_{\mu, i}-\widehat{\phi}_{\nu} V_{\mu, \nu}\right) .
$$

On the other hand, according to Equation (13) and Proposition 21, $\widehat{\phi}_{\nu} V_{\mu, \nu}$ is the least integral multiple of $s_{\nu}^{\mu}$, for which

$$
\widehat{\phi}_{\nu} V_{\mu, \nu} \geq M_{\nu}+\widehat{d}_{\nu} V_{\mu, \nu} \xi
$$

where the inequality is strict if $\widehat{d}_{\nu}=0$. Observe that Proposition 21 yields

$$
M_{\nu}+\widehat{d}_{\nu} V_{\mu, \nu} \xi=\sum_{j \in \Gamma_{\nu}^{\mu}}\left(v_{\Gamma}(j)-2\right) V_{\mu, j}+\widehat{d}_{\nu} V_{\mu, \nu} \xi=\left(\widehat{g}_{\langle\widehat{\kappa}\rangle}\right)_{\nu} V_{\mu, \nu},
$$

where the last equality follows from Lemma 13 . Since every $V_{\mu, i}$ is divisible by $s_{\nu}^{\mu}$ for $i \in \Gamma_{\nu}^{\mu}$, we see that $h_{\mu}^{\mathfrak{a}}(\xi) \in S^{\mu}$, if for every $\nu \sim \mu$ holds

$$
\sum_{i \in \Gamma_{\nu}^{\mu}} \widehat{f}_{i} V_{\mu, i} \geq\left(\widehat{g}_{\langle\widehat{\kappa}\rangle}\right)_{\nu} V_{\mu, \nu}
$$

Observe that in the case $\widehat{d}_{\nu}=0$ the right hand side is equal to $M_{\nu}$ which is not in $\mathcal{S} \mathcal{V}_{\nu}^{\mu}$ by Proposition 21, while the left hand side clearly is in $\mathcal{S} \mathcal{V}_{\nu}^{\mu}$. Thus the inequality must be strict in this case. 
As we saw above, $\widehat{g}^{\mathcal{N}}=\widehat{f}^{\mathcal{N}}$ and $\left(\widehat{g}_{\langle\widehat{\kappa}\rangle}\right)_{i}=0$ unless $d(\mu, i) \leq 1$. Therefore $\widehat{g}_{\langle\widehat{\kappa}\rangle}=\widehat{f}_{\left[\widehat{g}_{\langle\widehat{\kappa}\rangle}\right]}^{\mathcal{N}}$, and $\widehat{f}_{\langle\widehat{f}\rangle}=\widehat{f}_{\left[\widehat{f}_{\langle\widehat{f}\rangle}\right.}^{\mathcal{N}}$ follows from Remark 12, Recall that for any
$\nu \sim \mu$

$$
\lambda\left((\widehat{f} V)_{\nu}, \nu\right) \geq \xi=\lambda\left((\widehat{g} V)_{\nu}, \nu\right) .
$$

Hence, by applying Lemma 13 , we obtain for any $\nu \sim \mu$

$$
\lambda\left(\left(\widehat{f}_{\left[\widehat{f}_{\langle\widehat{f}\rangle}^{\mathcal{N}}\right]}^{\mathcal{N}} V\right)_{\nu}, \nu\right)=\lambda\left(\left(\widehat{f}_{\langle\widehat{f}\rangle} V\right)_{\nu}, \nu\right) \geq \lambda\left(\left(\widehat{g}_{\langle\widehat{\kappa}\rangle} V\right)_{\nu}, \nu\right)=\lambda\left(\left(\widehat{f}_{\left[\widehat{g}_{\langle\widehat{\kappa}\rangle}\right.}^{\mathcal{N}} V\right)_{\nu}, \nu\right),
$$

so that

$$
\begin{aligned}
& \left(\widehat{f}_{\left[\widehat{f}_{\langle\widehat{f}\rangle}^{\mathcal{N}}\right.}^{\mathcal{N}} V\right)_{\nu}=\left(\widehat{f}^{\mathcal{N}} V\right)_{\nu}+\sum_{i \sim \mu}\left(\widehat{f}_{\langle\widehat{f}\rangle}\right)_{i} \varphi_{\mu}^{[\mu, i]}(\nu) V_{\mu, \nu} \\
\geq & \left(\widehat{f}_{\left[\widehat{g}_{\langle\widehat{\kappa}\rangle}^{\mathcal{N}}\right]} V\right)_{\nu}=\left(\widehat{f}^{\mathcal{N}} V\right)_{\nu}+\sum_{i \sim \mu}\left(\widehat{g}_{\langle\widehat{\kappa}\rangle}\right)_{i} \varphi_{\mu}^{[\mu, i]}(\nu) V_{\mu, \nu}
\end{aligned}
$$

According to Proposition $11 \varphi_{\mu}^{[\mu, i]}(\nu) \geq 0$ for $\nu \sim \mu$, where the equality takes place always if $\mu \sim i \neq \nu$. Subsequently,

$$
\left(\widehat{f}_{\langle\widehat{f}\rangle}\right)_{\nu} \varphi_{\mu}^{[\mu, \nu]}(\nu) V_{\mu, \nu} \geq\left(\widehat{g}_{\langle\widehat{\kappa}\rangle}\right)_{\nu} \varphi_{\mu}^{[\mu, \nu]}(\nu) V_{\mu, \nu}
$$

which yields Inequality (14). Thus $h_{\mu}^{\mathfrak{a}}(\xi) \in S^{\mu}$.

Suppose next that $h_{\mu}^{\mathfrak{a}}(\xi) \in S^{\mu}$. Then we may take such non-negative integers $m_{\nu}$ for $\nu \sim \mu$, that

$$
h_{\mu}^{\mathfrak{a}}(\xi)=\sum_{\nu \sim \mu} m_{\nu} s_{\nu}^{\mu}
$$

Let $w:=\sum_{\nu \sim \mu}\left(m_{\nu} s_{\nu}^{\mu} / V_{\mu, \nu}\right) \mathbf{1}_{\nu}$ and define $\widehat{\psi}:=\widehat{\phi}_{[w]}$. Subsequently, we obtain by using Equation (12) and Lemma 13

$$
\widehat{\psi}_{\mu}=\frac{h_{\mu}^{\mathfrak{a}}(\xi)}{V_{\mu, \mu}}-\sum_{\nu \sim \mu} \frac{m_{\nu} s_{\nu}^{\mu}}{V_{\mu, \nu}} \frac{V_{\mu, \nu}}{V_{\mu, \mu}}=0,
$$

while for $\nu \sim \mu$ Equation (13) together with Lemma 13 yields

$$
\widehat{\psi}_{\nu}=\frac{s_{\nu}^{\mu}}{V_{\mu, \nu}}\left\lceil\frac{\widehat{d}_{\nu} V_{\mu, \nu}}{s_{\nu}^{\mu}} \xi\right]^{+}+\frac{M_{\nu}}{V_{\mu, \nu}}+\frac{m_{\nu} s_{\nu}^{\mu}}{V_{\mu, \nu}}
$$

Clearly, $\widehat{\psi}_{\nu}=0$ unless $\nu \sim \mu$, and further, since $s_{\nu}^{\mu}$ divides $\widehat{\psi}_{\nu} V_{\mu, \nu}$ and $\widehat{\psi}_{\nu} V_{\mu, \nu}>$ $M_{\nu}$ we observe by Lemma 21 that $\widehat{\psi}_{\nu} V_{\mu, \nu} \in \mathcal{S} \mathcal{V}_{\nu}^{\mu}$ for every $\nu \sim \mu$. Therefore we may find $\widehat{f} \in \mathbb{N}^{\Gamma}$ with $\hat{f}_{i}>0$ only if $v_{\Gamma}(i)=1$ and $i \neq \mu$ satisfying

$$
\left(\widehat{f}_{\langle\widehat{f}\rangle}\right)_{\nu}=\widehat{\psi}_{\nu}
$$

for every $\nu \sim \mu$. It follows from Lemma 13 that for any $\nu$ with $d(\mu, \nu) \leq 1$

$$
\lambda\left((\widehat{f} V)_{\nu}, \nu\right)=\lambda\left((\widehat{\psi} V)_{\nu}, \nu\right) \geq \lambda\left((\widehat{\phi} V)_{\nu}, \nu\right) \geq \xi,
$$

where the equality holds for $\nu=\mu$ and otherwise it may take place only if $\widehat{d}_{\nu}>0$. Subsequently, by choosing $a_{i}=\widehat{f} V_{i}$ for every $i$ with $d(i, \mu) \leq 1$, we 
may by using [6, Lemma 5 and Lemma 6] achieve a connected set $U \subset\{\nu \in$ $\Gamma \mid d(\nu, \mu) \leq 1\}$ and non-negative integers $a_{i}$ for every $i \in \Gamma$ with $d(i, U) \leq 1$ satisfying the conditions of [6, Theorem 1]. Thereby $\xi \in \mathcal{H}_{\mu}^{\mathfrak{a}}$.

\section{EXAMPLES}

Cases of low valence.

Example 27. In the case $v_{\Gamma}(\mu)=0$ we have only one vertex $\mu$. Moreover, $\widehat{d}_{\mu}>0$ while $V_{\mu, \mu}=1$ and thereby $d_{\mu}=\widehat{d}_{\mu}$. As the set of vertices adjacent to $\mu$ is empty, the set $S^{\mu}$ is $\mathbb{N}$. The claim of Theorem 23 now says that $\xi$ is a jumping number if and only if

$$
\widehat{d}_{\mu} \xi-2 \in \mathbb{N} \text {. }
$$

But this already follows from [8, Theorem 6.2] and Remark 17.

Example 28. Suppose $v_{\Gamma}(\mu)=1$. Let $\nu$ be the vertex adjacent to $\mu$. Then

$$
d_{\mu} \xi=\widehat{d}_{\mu} V_{\mu, \mu} \xi+\sum_{i \in \Gamma_{\nu}^{\mu}} s_{\nu}^{\mu} \frac{\widehat{d_{i}} V_{\mu, i}}{s_{\nu}^{\mu}} \xi
$$

Since $\left(v_{\Gamma}(\mu)-2\right) V_{\mu, \mu}=-V_{\mu, \mu}$, we get by Theorem 23

$$
h_{\mu}^{\mathfrak{a}}(\xi)=d_{\mu} \xi-V_{\mu, \mu}-s_{\nu}^{\mu}\left\lceil\sum_{i \in \Gamma_{\nu}^{\mu}} \frac{\widehat{d}_{i} V_{\mu, i}}{s_{\nu}^{\mu}} \xi\right]^{+}
$$

Putting these together shows that

$$
h_{\mu}^{\mathfrak{a}}(\xi)=\left(\widehat{d}_{\mu} \xi-1\right) V_{\mu, \mu}+s_{\nu}^{\mu} \sum_{i \in \Gamma_{\nu}^{\mu}} \frac{\widehat{d}_{i} V_{\mu, i}}{s_{\nu}^{\mu}} \xi-s_{\nu}^{\mu}\left\lceil\sum_{i \in \Gamma_{\nu}^{\mu}} \frac{\widehat{d}_{i} V_{\mu, i}}{s_{\nu}^{\mu}} \xi\right]^{+}<0
$$

always unless $\widehat{d}_{\mu} \xi \geq 1$. Especially, $\mathcal{H}_{\mu}^{\mathfrak{a}}$ is empty if $\widehat{d}_{\mu}=0$.

Example 29. Suppose $v_{\Gamma}(\mu)=2$. Since $\left(v_{\Gamma}(\mu)-2\right) V_{\mu, \mu}=0$, we get

$$
\begin{aligned}
h_{\mu}^{\mathfrak{a}}(\xi) & =d_{\mu} \xi-\sum_{\nu \sim \mu} s_{\nu}^{\mu}\left[\sum_{i \in \Gamma_{\nu}^{\mu}} \frac{\widehat{d}_{i} V_{\mu, i}}{s_{\nu}^{\mu} \xi}\right]^{+} \\
& =\sum_{i \in \Gamma} \widehat{d}_{i} V_{\mu, i} \xi-\sum_{\nu \sim \mu} s_{\nu}^{\mu}\left[\sum_{i \in \Gamma_{\nu}^{\mu}} \frac{\widehat{d}_{i} V_{\mu, i}}{s_{\nu}^{\mu}} \xi\right]^{+} \\
& =\widehat{d}_{\mu} V_{\mu, \mu} \xi+\sum_{\nu \sim \mu} \sum_{i \in \Gamma_{\nu}^{\mu}} \widehat{d}_{i} V_{\mu, i} \xi-\sum_{\nu \sim \mu} s_{\nu}^{\mu}\left[\sum_{i \in \Gamma_{\nu}^{\mu}} \frac{\widehat{d_{i}} V_{\mu, i}}{s_{\nu}^{\mu}} \xi\right]^{+} \\
& =\widehat{d}_{\mu} V_{\mu, \mu} \xi+\sum_{\nu \sim \mu} s_{\nu}^{\mu}\left(\sum_{i \in \Gamma_{\nu}^{\mu}} \frac{\widehat{d}_{i} V_{\mu, i}}{s_{\nu}^{\mu}} \xi-\left\lceil\sum_{i \in \Gamma_{\nu}^{\mu}} \frac{\widehat{d}_{i} V_{\mu, i}}{s_{\nu}^{\mu}} \xi\right]^{+}\right) .
\end{aligned}
$$


Suppose $\xi \in \mathcal{H}_{\mu}^{\mathfrak{a}}$. Then $h_{\mu}^{\mathfrak{a}}(\xi) \geq 0$, which implies that either $\widehat{d}_{\mu}>0$ or $\widehat{d}_{\mu}=0$ and

$$
\sum_{i \in \Gamma_{\nu}^{\mu}} \frac{\widehat{d}_{i} V_{\mu, i}}{s_{\nu}^{\mu}} \xi=\left\lceil\sum_{i \in \Gamma_{\nu}^{\mu}} \frac{\widehat{d}_{i} V_{\mu, i}}{s_{\nu}^{\mu}} \xi\right]^{+}
$$

for each $\nu \sim \mu$. Note that the latter is possible only if for each $\nu \sim \mu$ there is such $i \in \Gamma_{\nu}^{\mu}$ that $\widehat{d}_{i}>0$.

Let us then assume that $\widehat{d}_{\mu}=0$, and let $\widehat{f}$ be such that

$$
\lambda\left((\widehat{f} V)_{\eta}, \eta\right) \geq \lambda\left((\widehat{f} V)_{\mu}, \mu\right)=\xi .
$$

By Equation (3) we have $w_{\Gamma}(\mu) V_{\mu, i}=\sum_{\nu \sim \mu} V_{\nu, i}+\delta_{\mu, i}$ for every $i \in \Gamma$. Moreover, by Equations (2) and (4)

$$
w_{\Gamma}(\mu)\left(k_{\mu}+1\right)-\sum_{\nu \sim \mu}\left(k_{\nu}+1\right)=\widehat{k}_{\mu}=2-v_{\Gamma}(\mu)=0 .
$$

Thereby

$$
\begin{aligned}
\lambda\left((\widehat{f} V)_{\mu}, \mu\right) & =\frac{(\widehat{f} V)_{\mu}+k_{\mu}+1}{(\widehat{d} V)_{\mu}} \\
& =\frac{\left.w_{\Gamma}(\mu)\left((\widehat{f} V)_{\mu}+k_{\mu}+1\right)\right)}{w_{\Gamma}(\mu)(\widehat{d} V)_{\mu}} \\
& =\frac{\sum_{\nu \sim \mu}(\widehat{f} V)_{\nu}+\widehat{f}_{\mu}+\sum_{\nu \sim \mu}\left(k_{\nu}+1\right)}{\sum_{\nu \sim \mu}(\widehat{d} V)_{\nu}} \\
& =\frac{(\widehat{f} V)_{\nu_{1}}+k_{\nu_{1}}+1+\widehat{f}_{\mu}+(\widehat{f} V)_{\nu_{2}}+k_{\nu_{2}}+1}{(\widehat{d} V)_{\nu_{1}}}, \quad+\quad(\widehat{d} V)_{\nu_{2}}
\end{aligned}
$$

where $\nu_{1} \sim \mu \sim \nu_{2}$. Furthermore, since we may assume that

$$
\lambda\left((\widehat{f} V)_{\nu_{1}}, \nu_{1}\right) \leq \lambda\left((\widehat{f} V)_{\nu_{2}}, \nu_{2}\right),
$$

this shows that

$$
\lambda\left((\widehat{f} V)_{\nu_{1}}, \nu_{1}\right) \leq \lambda\left((\widehat{f} V)_{\mu}, \mu\right) \leq \lambda\left(\widehat{f}_{\mu}+(\widehat{f} V)_{\nu_{2}}, \nu_{2}\right),
$$

where the equality holds on the left if and only if it holds on the right. Putting these together with 15 we observe that $\widehat{f}_{\mu}=0$ and

$$
\lambda\left((\widehat{f} V)_{\nu_{1}}, \nu_{1}\right)=\xi=\lambda\left((\widehat{f} V)_{\nu_{2}}, \nu_{2}\right) .
$$

This is to say that both the vertices adjacent to $\mu$ support $\xi$. This means, informally speaking, that $\mu$ doesn't support jumping numbers independently. Especially, $\mathcal{H}_{\mu}^{\mathfrak{a}}$ is empty if there is $\nu$ such that $\widehat{d}_{i}=0$ whenever $i \in \Gamma_{\mu}^{\nu}$. 
Simple ideals.

Example 30. Suppose $\mathfrak{a}$ is a simple ideal. If $\mathfrak{a}$ is the maximal ideal, then the dual graph consists of one vertex, but this case is already discussed in Example 27. Thus we may assume $\mathfrak{a}$ is different from the maximal ideal. Since $\widehat{d_{i}}>0$ for only one vertex $i$, the examples 28 and 29 show that if $v_{\Gamma}(\mu)<3$ and $\widehat{d}_{\mu}=0$ then $\mathcal{H}_{\mu}^{\mathfrak{a}}$ is empty. Thus we may suppose that $\mu$ is a vertex with $\widehat{d}_{\mu}=1$ or $v_{\Gamma}(\mu)=3$. Note that if $\widehat{d}_{\mu}=1$, then $v_{\Gamma}(\mu)<3$ and if $v_{\Gamma}(\mu)=3$ then $\widehat{d}_{\mu}=0$.Let $(\gamma, \tau)$ be the pair associated to $\mu$.

Consider first the case $\widehat{d}_{\mu}=1$ and $v_{\Gamma}(\mu)=1$. Then $\Gamma_{\gamma}^{\mu}$ is the only branch emanating from $\mu$. By using Proposition 21 we obtain $s_{\gamma}^{\mu}=q_{\mu, \gamma}$, and by applying equation $P Q=1$, we see that $q_{\mu, \gamma}=q_{\mu, \mu}=1$. Thus $S^{\mu}=\mathbb{N}$, and by Theorem 23, $\xi \in \mathcal{H}_{\mu}^{\mathfrak{a}}$ if and only if

$$
(\xi-1) d_{\mu}-1 \in \mathbb{N} \text {, i. e., } \xi \in\left\{1+\frac{t+1}{d_{\mu}} \mid t \in \mathbb{N}\right\} .
$$

Consider next the case $\widehat{d}_{\mu}=1$ and $v_{\Gamma}(\mu)=2$. It follows from Proposition 21 that $s_{\gamma}^{\mu}=a:=q_{\mu, \gamma}$. Furthermore, $s_{\tau}^{\mu}=b:=V_{\mu, \tau}$ and $d_{\mu}=a b$. Now $S^{\mu}=\langle a, b\rangle$, and Theorem 23 says that $\xi \in \mathcal{H}_{\mu}^{\mathfrak{a}}$ if and only if

$$
a b \xi-a-b \in\langle a, b\rangle, \text { i. e., } \xi \in\left\{\frac{s+1}{a}+\frac{t+1}{b} \mid s, t \in \mathbb{N}\right\} \text {. }
$$

In the case $v_{\Gamma}(\mu)=3$ we see, again by Proposition 21, that $s_{\gamma}^{\mu}=a:=q_{\mu, \gamma}$ and $s_{\tau}^{\mu}=b:=V_{\mu, \tau}$ and $V_{\mu, \mu}=a b$, while $s_{\eta}^{\mu}=V_{\mu, \mu}$, where $\eta$ is the vertex corresponding to $\mathfrak{a}$. According to Theorem $23 \xi \in \mathcal{H}_{\mu}^{\mathfrak{a}}$ if and only if

$$
V_{\mu, \eta} \xi+V_{\mu, \mu}-a-b-V_{\mu, \mu}\left\lceil\frac{V_{\mu, \eta} \xi}{V_{\mu, \mu}}\right\rceil \in\langle a, b\rangle \text {. }
$$

Now $V_{\mu, \eta} / V_{\mu, \mu}=c:=q_{\eta, \mu}$, so the above is equivalent to

$$
\xi-\frac{\lceil c \xi\rceil-1}{c}=\frac{s+1}{a c}+\frac{t+1}{b c} \text { for some } s, t \in \mathbb{N} .
$$

Obviously, the equation holds for $\xi+\frac{1}{c}$ if it holds for $\xi$. Subsequently, $\xi \in \mathcal{H}_{\mu}^{\mathfrak{a}}$ if and only if

$$
\xi \in\left\{\frac{s+1}{a c}+\frac{t+1}{b c}+\frac{m}{c} \mid s, t, m \in \mathbb{N}, \frac{s+1}{a c}+\frac{t+1}{b c} \leq \frac{1}{c}\right\} .
$$

Observe that $a c=q_{\eta, \gamma}$ and $b c=V_{\mu, \tau} q_{\eta, \mu}=V_{\eta, \tau}$ by, e. g., [8, Proposition 3.13].

The above shows that Theorem 23 gives an alternative proof of the formula for jumping numbers of a simple ideal (see [8, Theorem 6.2]).

General case. 
Example 31. Let $\mathfrak{a}=\mathfrak{p}_{1}^{2} \mathfrak{p}_{2} \mathfrak{p}_{3}^{2} \mathfrak{p}_{4} \mathfrak{p}_{5}^{3}$ be an ideal, where

$$
\begin{aligned}
& \mathfrak{p}_{1}=\left\langle\overline{x^{3} y^{3}\left(x^{3}-y^{2}\right),\left(x^{3}-y^{2}\right)^{3}+x^{11}}\right\rangle, \\
& \mathfrak{p}_{2}=\left\langle\overline{x^{2} y^{3},\left(x^{3}-y^{2}\right)^{2}}\right\rangle, \\
& \mathfrak{p}_{3}=\left\langle\overline{x y^{5}, x^{3}-y^{7}}\right\rangle, \\
& \mathfrak{p}_{4}=\left\langle\overline{x^{10},\left(x^{3}-(x-y)^{2}\right)^{3}}\right\rangle, \\
& \mathfrak{p}_{5}=\left\langle\overline{y^{2}, x-y}\right\rangle .
\end{aligned}
$$

The dual graph of principalization of $\mathfrak{a}$ is as follows:

$\Gamma:$

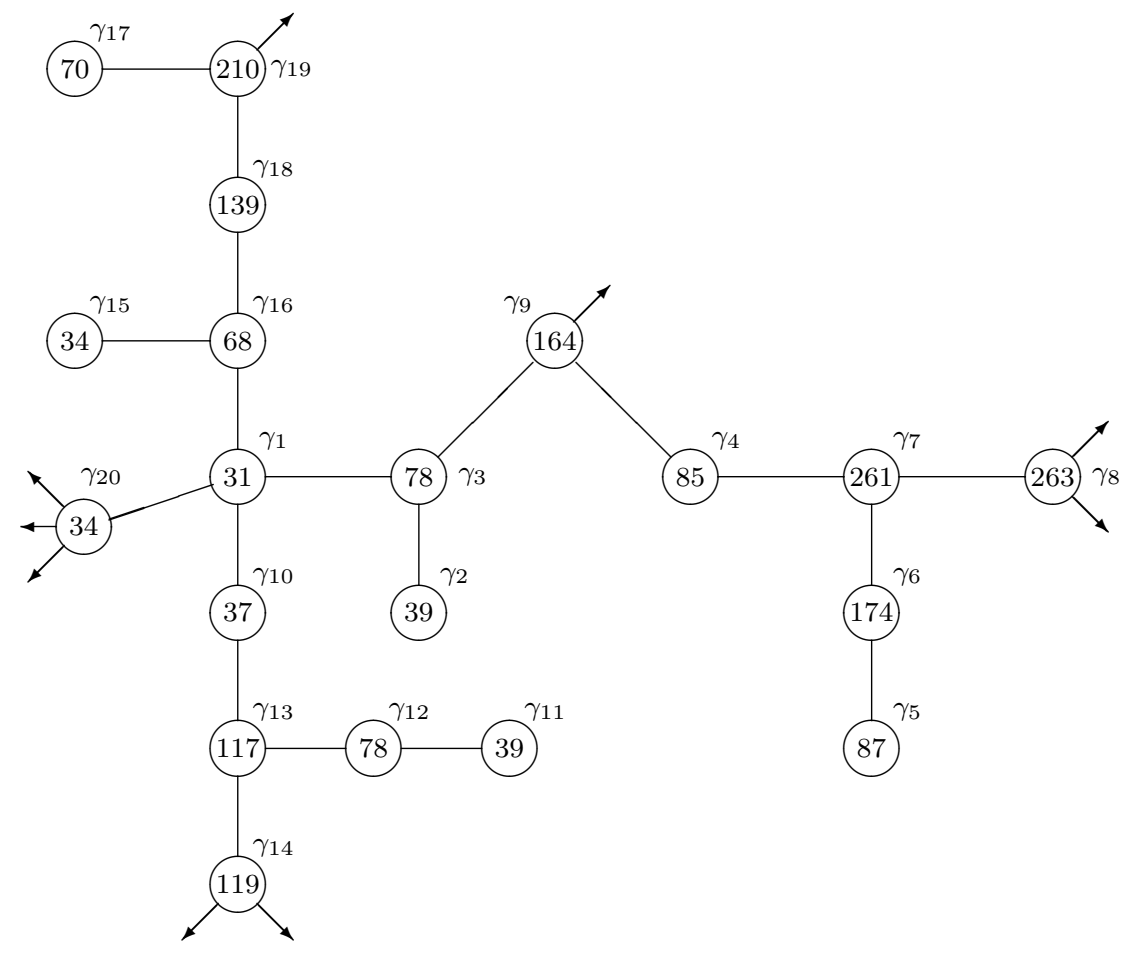

The factorization vector of $\mathfrak{a}$ is

$$
(0,0,0,0,0,0,0, \mathbf{2}, \mathbf{1}, 0,0,0,0, \mathbf{2}, 0,0,0,0, \mathbf{1}, \mathbf{3}),
$$

and 


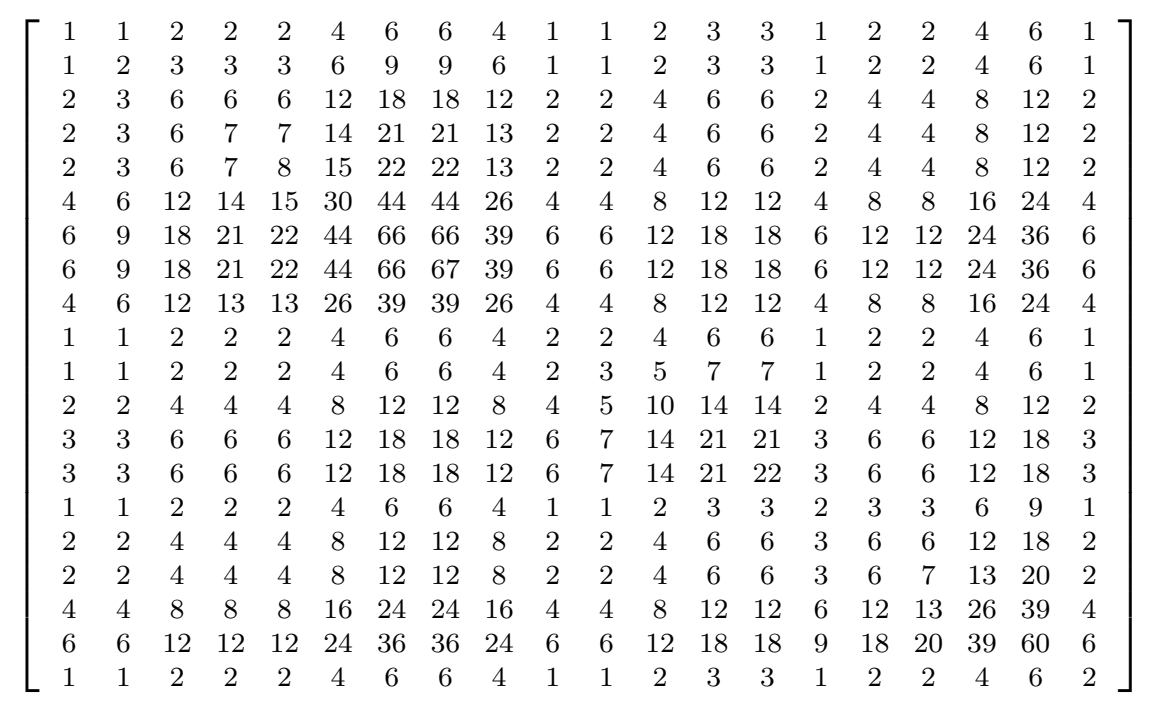

is the valuation matrix of $\mathfrak{a}$. Recall that by Proposition 9 any jumping number is supported at some vertex $\gamma$ with $\widehat{d}_{\gamma}>0$ or $v_{\Gamma}(\gamma)>2$. Therefore it is enough to consider the sets $\mathcal{H}_{\gamma_{j}}^{\mathfrak{a}}$ for $j=1,3,7,8,9,13,14,16,19$ and 20. By Theorem 23 we know that

$$
\mathcal{H}_{\gamma_{1}}^{\mathfrak{a}}=\left\{\frac{t}{31} \mid t+\left(v_{\Gamma}\left(\gamma_{1}\right)-2\right) V_{\gamma_{1}, \gamma_{1}}-\sum_{\nu \sim \gamma_{1}} s_{\nu}^{\gamma_{1}}\left\lceil t \sum_{i \in \Gamma_{\nu}^{\gamma_{1}}} \frac{\widehat{d}_{i} V_{\gamma_{1}, i}}{s_{\nu}^{\gamma_{1}} d_{\mu}}\right]^{+} \in S^{\gamma_{1}}\right\} .
$$

Clearly, $v_{\Gamma}\left(\gamma_{1}\right)=4$ and $V_{\gamma_{1}, \gamma_{1}}=1$. Furthermore, we have $s_{\nu}^{\gamma_{1}}=1$ for every $\nu \sim \gamma_{1}$ so that $S^{\gamma_{1}}=\mathbb{N}$, and if we write $\Psi_{\gamma, \nu}:=\sum_{i \in \Gamma_{\nu}^{\gamma}} \widehat{d}_{i} V_{\gamma, i}$, we see that

$$
\left(\Psi_{\gamma_{1}, \nu}\right)_{\nu=\gamma_{3}, \gamma_{10}, \gamma_{16}, \gamma_{20}}=(16,6,6,3) \text {. }
$$

Subsequently,

$$
\mathcal{H}_{\gamma_{1}}^{\mathfrak{a}}=\left\{\frac{t}{31} \mid t+2-\left\lceil\frac{16 \cdot t}{31}\right\rceil^{+}-2 \cdot\left\lceil\frac{6 \cdot t}{31}\right\rceil^{+}-\left\lceil\frac{3 \cdot t}{31}\right\rceil^{+} \geq 0\right\} .
$$

In the case $j=3$ we see that $v_{\Gamma}\left(\gamma_{3}\right)=3$ and $V_{\gamma_{3}, \gamma_{3}}=6$. Moreover, $\left(s_{\nu}^{\gamma_{3}}\right)_{\nu=\gamma_{1}, \gamma_{2}, \gamma_{9}}=(2,3,6)$ so that $S^{\gamma_{3}}=\langle 2,3\rangle$, and

$$
\left(\Psi_{\gamma_{3}, \nu}\right)_{\nu=\gamma_{1}, \gamma_{2}, \gamma_{9}}=(30,0,48) .
$$

Since $\lceil 0\rceil^{+}=1$ we see that

$$
\begin{aligned}
\mathcal{H}_{\gamma_{3}}^{\mathfrak{a}} & =\left\{\frac{t}{78} \mid t+6-2 \cdot\left\lceil\frac{30 \cdot t}{2 \cdot 78}\right]^{+}-3 \cdot\left\lceil\frac{0 \cdot t}{3 \cdot 78}\right]^{+}-6 \cdot\left\lceil\frac{48 \cdot t}{6 \cdot 78}\right]^{+} \in \mathbb{N} \backslash\{1\}\right\} \\
& =\left\{\frac{t}{78} \mid t+3-2 \cdot\left\lceil\frac{30 \cdot t}{2 \cdot 78}\right]^{+}-6 \cdot\left\lceil\frac{48 \cdot t}{6 \cdot 78}\right]^{+} \in \mathbb{N} \backslash\{1\}\right\} .
\end{aligned}
$$


Similarly,

$$
\begin{aligned}
\mathcal{H}_{\gamma_{7}}^{\mathfrak{a}}=\left\{\frac{t}{261} \mid t+44-3 \cdot\left[\frac{129 \cdot t}{3 \cdot 261}\right]^{+}-66 \cdot\left[\frac{132 \cdot t}{66 \cdot 261}\right]^{+} \in\langle 3,22\rangle\right\}, \\
\mathcal{H}_{\gamma_{8}}^{\mathfrak{a}}=\left\{\frac{t}{263} \mid t-67-\left[\frac{129 \cdot t}{263}\right]^{+} \geq 0\right\}, \\
\mathcal{H}_{\gamma_{9}}^{\mathfrak{a}}=\left\{\frac{t}{164} \mid t-2 \cdot\left[\frac{60 \cdot t}{2 \cdot 164}\right]^{+}-13 \cdot\left[\frac{78 \cdot t}{13 \cdot 164}\right]^{+} \in\langle 2,13\rangle\right\}, \\
\mathcal{H}_{\gamma_{13}}^{\mathfrak{a}}=\left\{\frac{t}{117} \mid t+14-3 \cdot\left[\frac{75 \cdot t}{3 \cdot 117}\right]^{+}-21 \cdot\left[\frac{42 \cdot t}{21 \cdot 117}\right]^{+} \in\langle 3,7\rangle\right\}, \\
\mathcal{H}_{\gamma_{14}^{\mathfrak{a}}}^{\mathfrak{a}}=\left\{\frac{t}{119} \mid t-22-\left[\frac{75 \cdot t}{119}\right]^{+} \geq 0\right\}, \\
\mathcal{H}_{\gamma_{16}}^{\mathfrak{a}}=\left\{\frac{t}{68} \mid t+3-2 \cdot\left[\frac{50 \cdot t}{2 \cdot 68}\right]^{+}-6 \cdot\left[\frac{18 \cdot t}{6 \cdot 68}\right]^{+} \in \mathbb{N} \backslash\{1\}\right\}, \\
\mathcal{H}_{\gamma_{19}}^{\mathfrak{a}}=\left\{\frac{t}{210} \mid t-20-3 \cdot\left[\frac{150 \cdot t}{3 \cdot 210}\right]^{+} \in\langle 3,20\rangle\right\},
\end{aligned}
$$

and finally,

$$
\mathcal{H}_{\gamma_{20}}^{\mathfrak{a}}=\left\{\frac{t}{34} \mid t-2-\left\lceil\left.\frac{28 \cdot t}{34}\right|^{+} \geq 0\right\}\right.
$$


Thus we get

$$
\begin{aligned}
\mathcal{H}_{\gamma_{1}}^{\mathfrak{a}} & =\left\{\frac{t+10 m}{31}+n \mid t=3,4,5,7,8,9,10 ; m=0,1,2\right\} \cup \mathbb{Z}_{+}, \\
\mathcal{H}_{\gamma_{3}}^{\mathfrak{a}} & =\left\{\frac{5+10 t+2 m}{78}+n \mid t, m, n \in \mathbb{N} ; t<8 ; m<3-\frac{t}{4}\right\} \cup \mathbb{Z}_{+}, \\
\mathcal{H}_{\gamma_{7}}^{\mathfrak{a}} & =\left\{\frac{t+3 m+129 p}{261}+n \mid t=46,89 ; m, n \in \mathbb{N} ; p=0,1 ; \frac{t+3 m+129 p}{261} \leq \frac{1+p}{2}\right\} \cup \mathbb{Z}_{+}, \\
\mathcal{H}_{\gamma_{8}}^{\mathfrak{a}} & =\left\{\frac{t+132}{263} \mid t \in \mathbb{N}\right\}, \\
\mathcal{H}_{\gamma_{9}}^{\mathfrak{a}} & =\left\{\frac{19+21 t+2 m}{164} \mid t, m \in \mathbb{N} \text { and } \frac{3-t}{3} \leq m \leq 4+\frac{16 t}{5}\right\}, \\
\mathcal{H}_{\gamma_{13}}^{\mathfrak{a}} & =\left\{\frac{t+3 m+57 p}{117}+n \mid t=22,41 ; m, n \in \mathbb{N} ; p=0,1 ; \frac{t+3 m+57 p}{117} \leq \frac{1+p}{2}\right\} \cup \mathbb{Z}_{+}, \\
\mathcal{H}_{\gamma_{14}}^{\mathfrak{a}} & =\left\{\frac{t+60}{119} \mid t \in \mathbb{N}\right\}, \\
\mathcal{H}_{\gamma_{16}}^{\mathfrak{a}} & =\left\{\frac{t+2 m}{68}+n \mid t=11,33,55,66 ; m=1,2,3,4,5,6 ; n \in \mathbb{N} \text { and } 23 \neq t+2 m \leq 68\right\}, \\
\mathcal{H}_{\gamma_{19}}^{\mathfrak{a}} & =\left\{\frac{t+3 m}{210} \mid t=71,142,210 ; m \in \mathbb{N}\right\}, \\
\mathcal{H}_{\gamma_{20}}^{\mathfrak{a}} & =\left\{\frac{t+12}{34} \mid t \in \mathbb{N}\right\},
\end{aligned}
$$

and the set of jumping numbers of $\mathfrak{a}$ is

$$
\mathcal{H}^{\mathfrak{a}}=\mathcal{H}_{\gamma_{1}}^{\mathfrak{a}} \cup \mathcal{H}_{\gamma_{3}}^{\mathfrak{a}} \cup \mathcal{H}_{\gamma_{7}}^{\mathfrak{a}} \cup \mathcal{H}_{\gamma_{8}}^{\mathfrak{a}} \cup \mathcal{H}_{\gamma_{9}}^{\mathfrak{a}} \cup \mathcal{H}_{\gamma_{13}}^{\mathfrak{a}} \cup \mathcal{H}_{\gamma_{14}}^{\mathfrak{a}} \cup \mathcal{H}_{\gamma_{16}}^{\mathfrak{a}} \cup \mathcal{H}_{\gamma_{19}}^{\mathfrak{a}} \cup \mathcal{H}_{\gamma_{20}}^{\mathfrak{a}}
$$

\section{REFERENCES}

[1] M. Alberich-Carramiñana, J. Àlvarez Montaner, F. Dachs-Cadefau, Multiplier ideals in two-dimensional local rings with rational singularities, Michigan Math. J. 65 (2016), no. 2, 287-320.

[2] A. Campillo, G. Gonzales-Sprinberg and M. Lejeune-Jalabert, Clusters of infinitely near points, Math. Ann. 306 (1996), 169-194.

[3] L. Ein, R. Lazarsfeld, K. Smith and D. Varolin, Jumping coefficients of multiplier ideals, Duke Math. J. 123 (2004), no. 3, 469-506.

[4] C. Galindo, Carlos, F. Monserrat, The Poincaré series of multiplier ideals of a simple complete ideal in a local ring of a smooth surface., Adv. Math. 225 (2010), no. 2, 1046-1068.

[5] C. Galindo, F. Hernando, F. Monserrat, The log-canonical threshold of a plane curve, Math. Proc. Cambridge Philos. Soc. 160 (2016), no. 3, 513-535.

[6] E. Hyry and T. Järvilehto, Jumping numbers and ordered tree structures on the dual graph, Manuscripta Math. 136 (2011), no. 3-4, 411-437.

[7] T. Järvilehto, Jumping numbers of a simple complete ideal in a two-dimensional regular local ring, $\mathrm{PhD}$ dissertation, University of Helsinki, 2007. 
[8] T. Järvilehto, Jumping numbers of a simple complete ideal in a two-dimensional regular local ring, Mem. Amer. Math. Soc. 214 (2011), no. 1009, viii+78 pp. ISBN: 978-0-82184811-1.

[9] T. Kuwata, On log canonical thresholds of reducible plane curves, Amer. J. Math. 121 (1999), no. 4, 701-721.

[10] J. Lipman, Proximity inequalities for complete ideals in two-dimensional regular local rings, Contemp. Math. 159 (1994), 293-306.

[11] J. Lipman and K. Watanabe, Integrally closed ideals in two-dimensional regular local rings are multiplier ideals, Math. Res. Lett. 10 (2003), no. 4, 423-434.

[12] D. Naie, Jumping numbers of a unibranch curve on a smooth surface, Manuscripta Math. 128 (2009), no. 1, 33-49.

[13] K. Smith and H. Thompson, Irrelevant exceptional divisors for curves on a smooth surface, Algebra, geometry and their interactions, Contemp. Math. 448 (2007), 245254.

[14] M. Spivakovsky, Valuations in function fields of surfaces, Amer. J. Math. 112 (1990), $107-156$.

[15] K. Tucker, Jumping numbers and multiplier ideals on algebraic surfaces, $\mathrm{PhD}$ dissertation, University of Michigan, 2010.

[16] K. Tucker, Jumping numbers on algebraic surfaces with rational singularities., Trans. Amer. Math. Soc. 362 (2010), no. 6, 3223-3241.

Mathematics and Statistics, Faculty of Natural Sciences, University of TAmpere, FIN-33014 TAmpereen yliopisto, Finland

Email address: eero.hyry@uta.fi

PÄÄSKYKUJA 5, FI-04620 MäNtsälä, Finland

Email address: tarmo.jarvilehto@pp.inet.fi 